\title{
HILBERT-KUNZ DENSITY FUNCTIONS AND F-THRESHOLDS
}

\author{
VIJAYLAXMI TRIVEDI AND KEI-ICHI WATANABE
}

\begin{abstract}
We had shown earlier that for a standard graded ring $R$ and a graded ideal $I$ in characteristic $p>0$, with $\ell(R / I)<\infty$, there exists a compactly supported continuous function $f_{R, I}$ whose Riemann integral is the HK multiplicity $e_{H K}(R, I)$. We explore further some other invariants, namely the shape of the graph of $f_{R, \mathbf{m}}$ (where $\mathbf{m}$ is the graded maximal ideal of $R$ ) and the maximum support (denoted as $\alpha(R, I))$ of $f_{R, I}$

In case $R$ is a domain of dimension $d \geq 2$, we prove that $(R, \mathbf{m})$ is a regular ring if and only if $f_{R, \mathbf{m}}$ has a symmetry $f_{R, \mathbf{m}}(x)=f_{R, \mathbf{m}}(d-x)$, for all $x$.

If $R$ is strongly $F$-regular on the punctured spectrum then we prove that the $F$-threshold $c^{I}(\mathbf{m})$ coincides with $\alpha(R, I)$.

As a consequence, if $R$ is a two dimensional domain and $I$ is generated by homogeneous elements of the same degree, then we have (1) a formula for the $F$-threshold $c^{I}(\mathbf{m})$ in terms of the minimum strong Harder-Narasimahan slope of the syzygy bundle and (2) a well defined notion of the $F$-threshold $c^{I}(\mathbf{m})$ in characteristic 0 .

This characterisation readily computes $c^{I(n)}(\mathbf{m})$, for the set of all irreducible plane trinomials $k[x, y, z] /(h)$, where $\mathbf{m}=(x, y, z)$ and $I(n)=\left(x^{n}, y^{n}, z^{n}\right)$.
\end{abstract}

\section{INTRODUCTION}

Let $(R, I)$ be a standard graded pair, i.e., $R$ is a Noetherian standard graded ring over a perfect field $k$ (unless otherwise stated) of characteristic $p>0$ and $I$ is a graded ideal of finite colength. Let $\mathbf{m}$ be the graded maximal ideal of $R$.

If $M$ is a finitely generated graded $R$-module then we have a compactly supported continuous function $f_{M, I}:[0, \infty) \longrightarrow[0, \infty)$ called the Hilbert-Kunz density function (see [T2]) for $(M, I)$ (we henceforth abbreviate the term 'Hilbert-Kunz' to the term 'HK'). We realize this function as the limit of a uniformly convergent sequence of compactly supported functions $\left\{f_{n}(M, I): \mathbb{R} \rightarrow[0, \infty)\right\}_{n \in \mathbb{N}}$, where

$$
f_{n}(M, I)(x)=\frac{1}{q^{d-1}} \ell\left(\left(M / I^{[q]} M\right)_{\lfloor x q\rfloor}\right) \text {, for } q=p^{n} .
$$

Moreover

$$
\int_{0}^{\infty} f_{M, I}(x) d x=e_{H K}(M, I)
$$

where $e_{H K}(M, I)$ denotes the famous invariant (introduced by P. Monsky [M1]) called the HK multiplicity of $M$ with respect to $I$.

Since the function $f_{M, I}$ is the uniformly convergent limit of the sequence $\left\{f_{n}(M, I)\right\}_{n}$, and is also an additive and a multiplicative function (the HK density functions of the rings explicitly gives the HK density function of their Segre product), it is a versatile tool to handle $e_{H K}(M, I)$.

In this paper we study the shape of the graph of $f_{R, I}$, and the maximal support $\alpha(R, I)$ of $f_{R, I}$, where

$$
\alpha(R, I)=\operatorname{Sup}\left\{x \mid f_{R, I}(x) \neq 0\right\} .
$$


First we prove (in Theorem 4.13 and Theorem 4.14) that the shape of the graph of $f_{R, \mathbf{m}}$ (and also the invariant $\left.\alpha(R, I)\right)$ determines the regularity of $(R, \mathbf{m})$ :

Theorem A. If $(R, \mathbf{m})$ is a standard graded domain of dimension $d \geq 2$ then

(1) $f_{R, \mathbf{m}}(x)=f_{R, \mathbf{m}}(d-x)$, for all $x$ if and only if the ring $(R, \mathbf{m})$ is regular.

(2) In fact $\alpha(R, \mathbf{m})=d$ if and only if $(R, \mathbf{m})$ is a regular ring.

(3) If $\operatorname{dim} R=2$ then either

(a) $f_{R, \mathbf{m}}$ is symmetric, i.e., $f_{R, \mathbf{m}}(1-y)=f_{R, \mathbf{m}}(1+y)$, for all $y \in(0,1)$, or

(b) $f_{R, \mathbf{m}}(1-y)>f_{R, \mathbf{m}}(1+y)$, for all $y \in(0,1)$.

Next we relate the invariant $\alpha(R, I)$ to $c^{I}(\mathbf{m})$, the $F$-threshold of $\mathbf{m}$ with respect to the ideal $I$.

Recall that the $F$-thresholds were introduced and studied in [MTW], in the case of regular rings. In a more general setting (when $R$ is not regular) it was further studied in [HMTW]. In [MTW] (Question 1.4) the following question was posed.

Question. Is it true that for all nonzero ideals $J$ and $I$ with $J \subseteq \operatorname{Rad}(I) \subseteq \mathbf{m}$, the $F$-threshold $c^{I}(J)$ is a rational number?

For regular rings, one gets a positive answer from a series of papers ([KLZ], [BMS1], [BMS2]) in the following way: the $F$-thresholds of $I$ are also $F$-jumping numbers of $I$, and all $F$-jumping numbers are rational.

Moreover if $R$ is a direct summand of a regular $F$-finite domain $S$, then, by Proposition 4.17 of $[\mathrm{AHN}], c^{I}(J)$ is a rational number. Here $c^{I}(J)$ was identified with $c^{I S}(J S)$ and hence is an $F$-jumping number of $J S$. We recall that in case $(R, \mathbf{m})$ is regular, either local or standard graded, and $J \subset R$ an ideal, then $c^{\mathbf{m}}(J)$ is the first jumping number $\operatorname{fpt}(J)$ (this is called the $F$-pure threshold of $J$ ).

However, in singular cases, $F$-thresholds may differ from $F$-jumping numbers, for example (1) when $R$ is the coordinate ring of the Segre product $\mathbb{P}^{m} \times \mathbb{P}^{n}$, where $m \neq n$, we have $\operatorname{fpt}(\mathbf{m})<c^{\mathbf{m}}(\mathbf{m})$ (see $[\mathrm{CM}]$ and [HWY]), (2) when $R=k[x, y, z] /\left(x y-z^{2}\right)$, we have $\operatorname{fpt}(\mathbf{m})=1<c^{\mathbf{m}}(\mathbf{m})=3 / 2$ (see [TW] and [HMTW]).

In general, to the best of our knowledge, it is not known whether $c^{\mathbf{m}}(\mathbf{m})$ is rational, even in graded cases.

As a consequence of identifying $c^{I}(\mathbf{m})$ with $\alpha(R, I)$ we prove that the $F$-thresholds $c^{I}(\mathbf{m})$ are rational numbers in the cases listed in Theorem B below.

Theorem B. For a standard graded pair $(R, I)$, where $R$ is a two dimensional domain, the following statements hold.

(1) If $I$ is generated by homogeneous elements of the same degree then $c^{I}(\mathbf{m})=$ $1-a_{\min }(V) / d$ and hence is a rational number.

(2) If $R$ is normal then $c^{I}(\mathbf{m})$ is a rational number.

Here $V$ denotes a syzygy bundle associated to the pair $(R, I)$ (as in Notations 6.2) and $a_{\text {min }}(V)$ denotes the minimum strong HN slope (as in Notations 5.3) and $d=e_{0}(R, \mathbf{m})$ denotes the Hilbert-Samuel multiplicity of $R$.

In another work [T5], the first author has used this explicit formulation of $c^{I}(\mathbf{m})$ in terms of the strong HN slopes of its syzygy bundle (along with a construction of D. Gieseker [G]) to give an example of a set of $F$-thresholds of an ideal with accumulation points, which answers another question by Mustaţă-Takagi-Watanabe (Question 2.11 in $[\mathrm{MTW}])$. 
Next we look at the reduction $\bmod p$ behaviour of these $F$-thresholds. Recall that Theorem 3.4 and Proposition 3.8 of [HY] describe the behaviour of $c^{\mathbf{m}}(I)$ under reduction $\bmod p$ (note that in this case $c^{\mathbf{m}}(I)=\mathrm{fpt}_{\mathbf{m}}(I)$ ) as follows:

Theorem [HY]. If $R=A\left[X_{1}, \ldots, X_{d}\right]$ is a polynomial ring over $A$, where $A$ is a localization of $\mathbb{Z}$ at some nonzero integer and $I \subset \mathbf{m}=\left(X_{1}, \ldots, X_{d}\right)$ is an ideal then

(1) $\lim _{p \rightarrow \infty} c^{\mathbf{m}_{p}}\left(I_{p}\right)$ exists and $\lim _{p \rightarrow \infty} c^{\mathbf{m}_{p}}\left(I_{p}\right)=$ lct $_{\mathbf{m}}(I)$, where lct $_{\mathbf{m}}(I)$ is the log canonical threshold of $I_{\mathbb{Q}}$ at $\mathbf{m}_{\mathbb{Q}}$.

(2) Moreover for $p>>0$, we have lct $\mathbf{m}_{\mathbf{m}}(I)=\lim _{p \rightarrow \infty} c^{\mathbf{m}_{p}}\left(I_{p}\right) \geq c^{\mathbf{m}_{p}}\left(I_{p}\right)$.

In dimension two, the formulation of $c^{I}(\mathbf{m})$ in terms of the strong HN slopes of a syzygy bundle (as in Theorem B) gives

(1) a well defined notion of $F$-threshold in characteristic 0 and

(2) a characterization of the strong semistability behaviour of the syzygy bundle $V_{s}$ (reduction $\bmod p_{s}$ of $V$ ) in terms of the $F$-threshold $c^{I_{s}}\left(\mathbf{m}_{s}\right)$, of $\mathbf{m}_{s}$ with respect to $I_{s}$ (see Definition 6.4).

This is done using the following result (proved in subsection 6.3):

Theorem C. Let $(R, I)$ be a standard graded pair where $R$ is a two dimensional domain in characteristic 0 and where $I$ is generated by homogeneous elements of the same degree. If $\left(R_{s}, I_{s}\right)$ is the reduction $\bmod p_{s}$ of the pair $(R, I)$, (obtained from a spread $\left(A, R_{A}, I_{A}\right)$, where $p_{s}=$ char $\left.R_{s}\right)$, then

$$
c_{\infty}^{I}(\mathbf{m}):=\lim _{p_{s} \rightarrow \infty} c^{I_{s}}\left(\mathbf{m}_{s}\right) \quad \text { exists and }
$$

(2) $c_{\infty}^{I}(\mathbf{m})=\operatorname{Sup}\left\{x \mid f_{R, I}^{\infty}(x) \neq 0\right\}$, where $f_{R, I}^{\infty}(x)=\lim _{p_{s} \rightarrow \infty} f_{R_{s}, I_{s}}(x)$.

(3) $c^{I_{s}}\left(\mathbf{m}_{s}\right) \geq c_{\infty}^{I}(\mathbf{m})$ if $p_{s}>>0$. If, in addition, the bundle $V$ is semistable and $V_{s}$ is the reduction $\bmod p_{s}$ of $V$ then

$$
c^{I_{s}}\left(\mathbf{m}_{s}\right)=c_{\infty}^{I}(\mathbf{m}) \Longleftrightarrow V_{s} \text { is strongly semistable. }
$$

In particular we have

Corollary D. Let $(R, \mathbf{m})$ be a standard graded pair, where $R$ is a two dimensional domain in characteristic 0 and let $X=\operatorname{Proj} R$ with $\operatorname{deg} \mathcal{O}_{X}(1)>2$ genus $(X)$, then for $p_{s}>>0$,

$$
c^{\mathbf{m}_{s}}\left(\mathbf{m}_{s}\right)=c_{\infty}^{\mathbf{m}}(\mathbf{m}) \Longleftrightarrow V_{s} \text { is strongly semistable. }
$$

We note that, in contrast to the Theorem $[\mathrm{HY}]$, here we have the following reverse inequality:

$$
\text { for } p_{s}>>0 \text { we have } c_{\infty}^{I}(\mathbf{m})=\lim _{p_{s} \rightarrow \infty} c^{I_{s}}\left(\mathbf{m}_{s}\right) \leq c^{I_{s}}\left(\mathbf{m}_{s}\right) .
$$

For higher dimensional cases, we relate the two invariants $c^{I}(\mathbf{m})$ and $\alpha(R, I)$ (in the subsection 4.1), which leads us to ask the following natural question

Question. Let $(R, I)$ be a standard graded pair and $\mathbf{m}$ be the graded maximal ideal of $R$. Then, is $\alpha(R, I)=c^{I}(\mathbf{m})$ ?

The following theorem (proved in the subsection 4.1) lists the cases where we show that the answer is affirmative

Theorem E. Let $(R, I)$ be a standard graded pair and $\mathbf{m}$ be the graded maximal ideal of $R$. Then

(1) $\alpha(R, I) \leq c^{I}(\mathbf{m})$, for dimension $R=d \geq 2$. Moreover 
(2) the equality $\alpha(R, I)=c^{I}(\mathbf{m})$ holds if the pair $(R, I)$ satisfies one of the following conditions:

(a) I is generated by a system of parameters,

(b) $R$ is strongly F-regular on the punctured spectrum $\operatorname{Spec} R \backslash\{\mathbf{m}\}$, or

(c) $R$ is a two dimensional domain and $I$ is generated by homogeneous elements of the same degree.

Moreover, the equality holds for the Segre products of all such pairs.

At the end of the paper, in Section 7, we give an explicit formula for $c^{I(n)}(\mathbf{m})$, where $R=k[x, y, z] /(h)$ are irreducible plane trinomials and where $I(n)=\left(x^{n}, y^{n}, z^{n}\right)$.

If we denote $c^{I(n)}(\mathbf{m})$ by $c^{I(n)_{p}}\left(\mathbf{m}_{p}\right)$ where $p=$ char $k$, then in fact we find that $c^{I(n)_{p}}\left(\mathbf{m}_{p}\right)$ (as $p$ varies) is just a function of the congruence class of $p \bmod 2 \lambda_{h}$, where $\lambda_{h}$ is an explicit computable integer given in terms of the exponents of the trinomial $h$ (see Notations 7.2). In particular we have the following

Example. If $R=k[x, y, z] /(h)$ is an irreducible trinomial of degree $d \geq 3$ then for all $p \geq d^{2}$ and $p \equiv \pm 1 \quad\left(\bmod 2 \lambda_{h}\right)$ we have $c^{I(n)_{p}}\left(\mathbf{m}_{p}\right)=c_{\infty}^{I(n)}(\mathbf{m})$.

If $(R, \mathbf{m})$ is a Segre product of any finitely many irreducible trinomials then

(1) there are infinitely many primes $p>0$, for which $c^{I(n)_{p}}\left(\mathbf{m}_{p}\right)=c_{\infty}^{I(n)}(\mathbf{m})$.

(2) Moreover, if one of the trinomials, occuring in the product, is a symmetric curve (i.e., $h=x^{d-a} y^{a}+y^{d-a} z^{a}+z^{d-a} x^{a}$ ) of degree $d>5$ then there are also infinitely many primes $p>0$, for which $c^{\mathbf{m}_{p}}\left(\mathbf{m}_{p}\right)>c_{\infty}^{\mathbf{m}}(\mathbf{m})$.

We recall the known computations made for some explicit polynomials to demonstrate the complexity of $c^{I}(J)$.

When $R=k[x, y]$ and $f=x^{2}+y^{3}$, or when $R=k[x, y, z]$ and $f$ is a homogeneous polynomial of degree 3 with isolated singularity at $(x, y, z)$ then $c^{\mathbf{m}}(f)$ was computed and such phenomena were exhibited in Examples 4.3 and 4.6 of [MTW]. In Corollary 3.9, Hara and Monsky (see $[\mathrm{H}]$ ) independently described (using sygygy gaps) the possible values of $c^{(x, y)}(f)$, whenever $f \in k[x, y]$ is homogeneous of degree 5 with an isolated singularity at the origin, when $p \neq 5$. Theorem 4.2 of $[\mathrm{Vr}] \operatorname{computes} c^{\mathbf{m}}(\mathbf{m})$, for diagonal hypersurfaces.

The organization of this paper is as follows.

In Section 3, we compute the HK density function for $(R, I)$, where $I$ is generated by a system of parameters. This turns out to be a volume function, depending only on the degrees of the generators of $I$. Here we use the uniform convergence property of the sequence $\left\{f_{n}(R, I)\right\}_{n}$ and the fact that $f_{R, I}$ is a continuous function.

In Section 4 , we relate $\alpha(R, I)$ with the $F$-threshold $c^{I}(\mathbf{m})$ and give the examples of the cases when the equality $\alpha(R, I)=c^{I}(\mathbf{m})$ does hold. Here we also characterize the regularity property of the ring $R$ in terms of the shape of the graph of $f_{R, \mathbf{m}}$ and also in terms of the number $\alpha(R, \mathbf{m})$.

From Sections 5 onwards we restrict to standard graded pairs in dimension two. In Section 5, we list some (known) results about vector bundles over nonsingular projective curves, which we use later in this paper.

In Section 6 , we give a notion (analogous to $f_{R, I}$ ) of the HK density function $f_{V, \mathcal{O}_{X}(1)}$, for a pair $\left(V, \mathcal{O}_{X}(1)\right)$, where $V$ is a vector-bundle on a nonsingular curve $X$ and $\mathcal{O}_{X}(1)$ is a very ample line-bundle on $X$. Then we relate $f_{R, I}$ with the HK density functions of the syzygy vector bundles which are expressed in terms ot their strong HN datum.

In Section 7, we give the computations of $F$-thresholds for plane trinomials. 
The results stated in Theorem 6.7 can be generalized by removing the hypothesis that the ideal $I$ be generated by homogeneous elements of same degree elements. However the arguments are technical and will appear in a subsequent paper.

The authors thank the referee for careful reading of the manuscript and providing various suggestions which greatly improved the exposition of the paper.

\section{PRELIMINARIES}

Let $(R, I)$ be a standard graded pair over a perfect field of characteristic $p>0$. Let $M$ be a finitely generated graded $R$-module. We recall the following known properties of $f_{M, I}$ from [T2].

(1) Additive property: Like HK multiplicity, the HK density function too have the additive property, which reduces the theory of $f_{M, I}$ to the theory of $f_{R, I}$, where $R$ is a normal domain: Let $\Lambda$ be the set of minimal prime ideals $P$ of $R$ such that $\operatorname{dim} R / P=\operatorname{dim} R$. Then

$$
f_{M, I}=\sum_{P \in \Lambda} f_{R / P, I} \lambda\left(M_{P}\right)
$$

As a consequence, we have

(a) $f_{M, I}=0$, if $\operatorname{dim} M<\operatorname{dim} R$.

(b) If $R$ is an integral domain then $f_{R, I}=f_{S, I S}$, where $S$ is the normalization of $R$, regarded as a graded $R$-module.

(c) $f_{M, I}=f_{M(n), I}$, for every $n \in \mathbb{Z}$.

(2) Multiplicative property: The multiplicative property expresses the HK density function of the Segre product of rings in terms of the HK density function of the individual rings: If $(R, I)$ and $(S, J)$ are two pairs and $F_{R}(x)=$ $e(R) x^{d-1}(d-1)$ !, where $e(R)$ denotes the Hilbert-Samuel multiplicity of $R$ with respect to its irrelevant maximal ideal $\mathbf{m}$ and $d=\operatorname{dim} R$ then the Segre product $(R \# S, I \# J)$ satisfies

$$
F_{R \# S}(x)-f_{R \# S, I \# J}(x)=\left[F_{R}(x)-f_{R, I}(x)\right]\left[F_{S}(x)-f_{S, J}(x)\right] .
$$

(3) Let $I \subseteq I^{\prime}$ such that $I^{\prime}$ is homogeneous then

$$
e_{H K}(R, I)=e_{H K}\left(R, I^{\prime}\right) \Longleftrightarrow f_{R, I}(x)=f_{R, I^{\prime}}(x) \text {, for all } x \text {. }
$$

In particular, if $R$ is equidimensional then

$$
f_{R, I}=f_{R, I^{\prime}} \Longleftrightarrow I^{\prime} \subseteq I^{*}
$$

where $I^{*}$ denotes the tight closure of $I$ in $R$.

(4) If $n_{0} \in \mathbb{N}$ such that $\mathbf{m}^{n_{0}} \subseteq I$ and the ideal $I$ is generated by $\mu$ generators then the support of $f_{R, I} \subseteq\left[0, n_{0} \mu\right]$.

\section{HK DENSITY FUNCTIONS FOR PARAMETER IDEALS}

3.1. HK density functions for parameter ideals. Here we give an explicit formula for the HK density function $f_{R, I}$, when $I$ is generated by a system of parameters. As expected, we find that $f_{R, I}$ solely depends on the degrees of the generators of $I$.

Definition 3.1. Given nonnegative integers $n_{1}, \ldots, n_{m}$, consider a $m$-parallelotope $P=\left[0, n_{1}\right] \times \cdots \times\left[0, n_{m}\right]$. We define a volume function

$$
V_{m-1}\left(n_{1}, \ldots, n_{m}\right):[0, \infty) \longrightarrow[0, \infty) \text { given by } x \rightarrow \operatorname{Vol}_{m-1}\left(P \cap H_{x}\right),
$$


where $H_{x}=\left\{\left(y_{1}, \ldots, y_{m}\right) \in \mathbb{R}^{m} \mid \sum_{i} y_{i}=x\right\}$ is a $m$-1-dimensional hyperplane in $\mathbb{R}^{m}$ and $\mathrm{Vol}_{m-1}$ is the $(m-1)$-dimensional Euclidean volume.

Lemma 3.2. Let $(R, I)$ be a standard graded pair, where $I$ is generated by homogeneous system of parameters $f_{1}, \ldots, f_{d}$ of degree $n_{1}, n_{2}, \ldots, n_{d}$ respectively. Then

$$
f_{R, I}(x)=e(R) V_{d-1}\left(n_{1}, \ldots, n_{d}\right)(x),
$$

where the function $V_{d-1}\left(n_{1}, \ldots, n_{d}\right)$ is given as in Definition 3.1 and $e(R)$ is the HilbertSamuel multiplicity of $R$ with respect to the ideal $\mathbf{m}$.

Proof. By the additive property of the HK density function and the Hilbert-Samuel multiplicity, we can assume that $R$ is a normal domain and hence $f_{1}$ is a non zerodivisor on $R$. For $d=2$ the lemma is easy to check as $\left\{f_{1}, f_{2}\right\}$ form a regular sequence. Henceforth we assume $d \geq 3$. We prove the lemma by induction on $d$. For the ring $S=R / f_{1} R$ and the ideal $J=I / f_{1} R$

$$
f_{S, J}(x)=e(S, \mathbf{m} S) V_{d-2}\left(n_{2}, \ldots, n_{d}\right)(x), \text { for all } x \in \mathbb{R},
$$

where, $e(S, \mathbf{m} S)=n_{1} e(R)$. For every $k \geq 1$ and $q=p^{n}$, there exists the canonical degree 0 surjective map of graded $R$-modules, where $J_{1}=f_{2} R+\cdots+f_{d} R$.

$$
\frac{S}{J[q]}\left(-k n_{1}\right) \longrightarrow \frac{f_{1}^{k} R}{f_{1}^{k+1} R+f_{1}^{k} R \cap J_{1}^{[q]}}=\frac{f_{1}^{k} R+J_{1}^{[q]}}{f_{1}^{k+1} R+J_{1}^{[q]}} .
$$

Hence, for any $x \geq 0$, we have the surjective map

$$
\oplus_{k=0}^{q-1}\left(\frac{S}{J^{[q]}}\right)_{-k n_{1}+\lfloor x q\rfloor} \longrightarrow\left(\frac{R}{I^{[q]}}\right)_{\lfloor x q\rfloor},
$$

which gives

$$
f_{n}(R, I)(x) \leq \frac{1}{q} \sum_{k=0}^{q-1} f_{n}(S, J)\left(\frac{\lfloor x q\rfloor-k n_{1}}{q}\right) .
$$

For the sake of brevity, throughout the rest of the proof, we denote $f_{n}(S, J)$ by $g_{n}$. For every $q=p^{n}, k \geq 1$ and for every $k n_{1} / q<\lambda \leq\left(k n_{1}+n_{1}\right) / q$ (applying Lemma 2.8 of [T2] to an injective graded map $\left.S_{\lfloor x q-\lambda q\rfloor} \longrightarrow S_{\lfloor x q\rfloor-k n_{1}}\right)$ we have

$$
g_{n}\left(\frac{\lfloor x q\rfloor-k n_{1}}{q}\right)=g_{n}\left(\frac{\lfloor x q\rfloor}{q}-\lambda\right)+O(1 / q) .
$$

Hence

$$
\left(\frac{1}{q}\right) g_{n}\left(\frac{\lfloor x q\rfloor-k n_{1}}{q}\right)=\frac{1}{n_{1}} \int_{k n_{1} / q}^{(k+1) n_{1} / q} g_{n}\left(\frac{\lfloor x q\rfloor}{q}-\lambda\right) d \lambda+O\left(1 / q^{2}\right)
$$

Case (1). If $n_{1}<x$. Then for $q>>0$, we have $(q-1) n_{1} \leq\lfloor x q\rfloor$.

Therefore

R.H.S. of $(\underline{3.2})=(1 / q) \sum_{k=0}^{q-1} g_{n}\left(\frac{\lfloor x q\rfloor-k n_{1}}{q}\right)=\left(1 / n_{1}\right) \int_{\lfloor x q\rfloor / q-n_{1}}^{\lfloor x q\rfloor / q} g_{n}(\lambda) d \lambda+O(1 / q)$.

Now taking limit for (3.2) as $q \rightarrow \infty$, and by induction on $d$, we get

$$
f_{R, I}(x) \leq\left(1 / n_{1}\right) \int_{x-n_{1}}^{x} f_{S, J}(\lambda) d \lambda=\frac{1}{n_{1}} e(S, \mathbf{m} S) \int_{x-n_{1}}^{x} V_{d-2}\left(n_{2}, \ldots, n_{d}\right)(\lambda) d \lambda .
$$

Since $n_{1}<x$, this gives $f_{R, I}(x) \leq e(R) V_{d-1}\left(n_{1}, n_{2}, \ldots, n_{d}\right)(x)$. 
Case (2) If $n_{1} \geq x$ then $\lfloor x q\rfloor=n_{1} \tilde{m}+r$, where $0 \leq r<n_{1}$ and $\tilde{m}<q-1$.

$$
\begin{aligned}
\text { R.H.S. of }(\underline{3.2})=(1 / q) & {\left[g_{n}\left(\frac{\lfloor x q\rfloor-\tilde{m} n_{1}}{q}\right)+g_{n}\left(\frac{\lfloor x q\rfloor-(\tilde{m}-1) n_{1}}{q}\right)+\cdots g_{n}\left(\frac{\lfloor x q\rfloor}{q}\right)\right] } \\
= & \left(1 / n_{1}\right) \int_{0}^{\tilde{m} n_{1} / q} g_{n}(\lambda) d \lambda+\tilde{m} O\left(1 / q^{2}\right) .
\end{aligned}
$$

Now taking limit for (3.2) as $q \rightarrow \infty$, we get

$$
f_{R, I}(x) \leq\left(1 / n_{1}\right) \int_{0}^{x} f_{S, J}(\lambda) d \lambda=e(R) V_{d-1}\left(n_{1}, \ldots, n_{d}\right)(x) .
$$

Hence $f_{R, I}(x) \leq e(R) V_{d-1}\left(n_{1}, \ldots, n_{d}\right)(x)$ for all $x \in \mathbb{R}$.

But $e(R) V_{d-1}\left(n_{1}, \ldots, n_{d}\right)(x)-f_{R, I}(x)$ is a nonnegative continuous function with integral $=0$. Therefore $f_{R, I}(x)=e(R) V_{d-1}\left(n_{1}, \ldots, n_{d}\right)(x)$, for all $x$.

Corollary 3.3. Let $(R, I)$ be a pair as above. If $I$ is a parameter ideal of $R$ generated by elements of degrees, say $n_{1}, \ldots, n_{d}$ then $f_{R, I}$ is a symmetric function around $n_{1}+$ $\cdots+n_{d}$, i.e.,

$$
f_{R, I}(x)=f_{R, I}\left(n_{1}+\cdots+n_{d}-x\right), \text { for all } x \geq 0 .
$$

4. The Function $f_{R, I}$ Versus the $F$-Threshold $c^{I}(\mathbf{m})$ AND the Regularity

4.1. Support of the HKd function. In this subsection we compare the maximum support of the HK density function $f_{R, I}$ and the $F$-threshold $c^{I}(\mathbf{m})$ of $\mathbf{m}$ with respect to $I$. We discuss the cases, where we can show that both the invariants coincide.

Definition 4.1. For a standard graded pair $(R, I)$ and a finitely generated graded $R$-module $M$, let

$$
\alpha(M, I)=\operatorname{Sup}\left\{x \mid f_{M, I}(x)>0\right\} .
$$

Remark 4.2. If $(R, I)$ is a standard graded pair of dimension $d \geq 2$ and $I$ is generated by homogeneous elements of degrees $d_{1} \leq d_{2}<\cdots$ then $\alpha(R, I)>d_{1}$ : By definition, the function $f_{R, I}(x)=e(R) x^{d-1} /\left((d-1)\right.$ !), for $0 \leq x \leq d_{1}$. In particular, $\left.f_{R, I}\right|_{\left[0, d_{1}\right]}$ is a strictly monotonic increasing function and $\alpha(R, I)$ is a positive real number with $\alpha(R, I)>d_{1}$.

We recall the following notion of $F$-threshold, as defined in [HMTW].

Definition 4.3. Let $I$ and $J$ be two ideals such that $J \subseteq \sqrt{I}$. Then the $F$-threshold of $J$ with respect to $I$ is

$$
c^{I}(J)=\lim _{q \rightarrow \infty} \frac{\min \left\{r \mid J^{r+1} \subseteq I^{[q]}\right\}}{q} \quad \text { if it exists. }
$$

The existence of the above limit in full generality was proved in [DsNbP].

Proposition 4.4. Let $(R, I)$ be a standard graded pair of dimension $\geq 2$. Then $\alpha(R, I) \leq c^{I}(\mathbf{m})$.

Proof. Let $c^{I}(\mathbf{m})=c$. Then, given $\epsilon>0$, there is a $q(\epsilon)$ such that for all $q \geq q(\epsilon)$, we have $\mathbf{m}^{(c+\epsilon) q} \subseteq I^{[q]}$. Since $R$ is a standard graded ring this implies $\left(R / I^{[q]}\right)_{m}=0$, for $m \geq(c+\epsilon) q$.

Therefore, for all $x \geq c+\epsilon$ and for $q \geq q(\epsilon)$,

$$
\ell\left(R / I^{[q]}\right)_{\lfloor x q\rfloor}=0 \Longrightarrow f_{n}(x)=\frac{1}{q^{d-1}} \ell\left(R / I^{[q]}\right)\lfloor x q\rfloor=0,
$$


Hence, for every $\epsilon>0, f(x)=\lim _{n \rightarrow \infty} f_{n}(x)=0$, for all $x \geq(c+\epsilon)$.

Since $f:[0, \infty) \longrightarrow[0, \infty)$ is a continuous function, we deduce that $f(x)=0$ for all $x \geq c$.

Next we discuss the cases where $\alpha(R, I)=c^{I}(\mathbf{m})$.

Lemma 4.5. For a standard graded pair $(R, I)$ of dimension 1 , where $R$ is a reduced ring, we have $\alpha(R, I)=c^{I}(\mathbf{m})$.

Proof. We recall (Theorem 2.9 [T2]) that $f_{R, I}$ is the pointwise limit of $f_{n}(R, I)$ (here the convergence may not be a uniform convergence). Moreover, for given $x \geq 0$, there is $n_{0}$ such that for all $q=p^{n} \geq p^{n_{0}}$, we have

$$
f_{R, I}(x)=f_{n}(R, I)(x)=\ell\left(R / I^{[q]}\right)_{\lfloor x q\rfloor} .
$$

Hence, for $x \geq \alpha(R, I)$,

$$
f_{R, I}(x)=0 \Longrightarrow \mathbf{m}^{\lfloor x q\rfloor} \subseteq I^{[q]} \text {, for } q \geq p^{n_{0}} \Longrightarrow c^{I}(\mathbf{m}) \leq x \Longrightarrow c^{I}(\mathbf{m})=\alpha(R, I) .
$$

Proposition 4.6. If $(R, I)$ is a standard graded pair where $R$ is a two dimensional domain and $I$ is generated by homogeneous elements of the same degree, then $\alpha(R, I)=$ $c^{I}(\mathbf{m})$.

Proof. After Proposition 4.4, we only need to prove $c^{I}(\mathbf{m}) \leq \alpha(R, I)$. Let $I$ be generated by homogeneous generators $h_{1}, \ldots, h_{\mu}$ all of degree $d_{0}$. By Proposition 2.12 of [T2], there is a constant $C_{0}$ such that for all $n \geq 0$ and for all $x \in[1, \infty)$ (here $q=p^{n}$ ),

$$
\left|f_{n}(x)-f_{n+1}(x)\right| \leq C_{0} / p^{n} .
$$

Therefore there is a constant $C_{1}$ such that

$$
\left|f_{n}(x)-f(x)\right| \leq C_{1} / p^{n} .
$$

Let $x_{0}=\alpha(R, I)$. Then, by Remark 4.2, we have $x_{0}>d_{0}\left(\right.$ note here $\left.q^{d-1}=q=p^{n}\right)$ and

$$
\frac{1}{p^{n}} \ell\left(R / I^{[q]}\right)_{\lfloor x q\rfloor}=f_{n}(x) \leq C_{1} / p^{n}, \text { for all } x \geq x_{0} \text { and } n \geq 0 .
$$

Therefore

$$
\ell\left(R / I^{[q]}\right)_{\lfloor x q\rfloor} \leq C_{1}, \text { for all } x \geq x_{0} \text { and } n \geq 0 .
$$

Let $X=$ Proj $R$. Let

$$
0 \longrightarrow V \longrightarrow \oplus^{\mu} \mathcal{O}_{X} \longrightarrow \mathcal{O}_{X}\left(d_{0}\right) \longrightarrow 0,
$$

be the short exact sequence of $\mathcal{O}_{X}$-sheaves, where $\oplus^{\mu} \mathcal{O}_{X} \longrightarrow \mathcal{O}_{X}\left(d_{0}\right)$ is the multiplication map given by $\left(a_{1}, \ldots, a_{\mu}\right) \rightarrow \sum_{i} h_{i} a_{i}$ (here $h_{i}$ are the chosen generators). This gives a long exact sequence of $\mathcal{O}_{X}$-modules, for every $m \in \mathbb{Z}$,

$$
\begin{gathered}
0 \longrightarrow H^{0}\left(X,\left(F^{n *} V\right)(m)\right) \longrightarrow \oplus H^{0}\left(X, \mathcal{O}_{X}(m)\right) \longrightarrow H^{0}\left(X, \mathcal{O}_{X}\left(m+q d_{0}\right)\right) \longrightarrow \\
\longrightarrow H^{1}\left(X,\left(F^{n *} V\right)(m)\right) \longrightarrow \oplus H^{1}\left(X, \mathcal{O}_{X}(m)\right) \longrightarrow \cdots .
\end{gathered}
$$

We can choose $m_{1} \geq 0$ such that, for all $m \geq m_{1}$,

$$
H^{1}\left(X, \mathcal{O}_{X}(m)\right)=0 \text { and } H^{0}\left(X, \mathcal{O}_{X}(m)\right)=R_{m} .
$$

In particular, for $m \geq m_{1}$,

$$
h^{1}\left(X,\left(F^{n *} V\right)(m)\right)=\ell\left(R / I^{[q]}\right)_{m+q d_{0}} .
$$


Since $\alpha(R, I)>d_{0}$ we can assume that $c^{I}(\mathbf{m})>d_{0}$ and therefore we can also choose $q_{0}$ large enough so that we have $\left(x_{0}-d_{0}\right) q_{0} \geq m_{1}$. Then for $q=p^{n} \geq q_{0}$,

$$
h^{1}\left(X,\left(F^{n *} V\right)(m)\right)=\ell\left(R / I^{[q]}\right)_{m+q d_{0}} \leq C_{1}, \text { for all } m \geq\left(x_{0}-d_{0}\right) q .
$$

We choose $z \in H^{0}\left(X, \mathcal{O}_{X}(1)\right)$ such that we have a short exact sequence of $\mathcal{O}_{X^{-}}$ modules

$$
0 \longrightarrow \mathcal{O}_{X}(-1) \longrightarrow \mathcal{O}_{X} \longrightarrow \mathcal{O}_{Y} \longrightarrow 0
$$

which induces the long exact sequence of $k$ vector-spaces

$$
\begin{gathered}
0 \longrightarrow H^{0}\left(X, F^{n *} V(m)\right) \longrightarrow H^{0}\left(X, F^{n *} V(m+1)\right) \longrightarrow H^{0}\left(X,\left.F^{n *} V(m+1)\right|_{Y}\right) \\
\longrightarrow H^{1}\left(X, F^{n *} V(m)\right) \longrightarrow H^{1}\left(X, F^{n *} V(m+1)\right) \longrightarrow 0,
\end{gathered}
$$

as $Y$ is a 0 -dimensional scheme, for every $m \in \mathbb{Z}$, we have $H^{1}\left(X,\left.F^{n *} V(m)\right|_{Y}\right)=0$ and $H^{0}\left(X,\left.F^{n *} V(m)\right|_{Y}\right)=C_{2}$, for some constant $C_{2}$.

Claim. $h^{1}\left(X, F^{n *} V(m)\right)=0$, for all $m \geq\left(x_{0}-d_{0}\right) q+C_{1}+1$, where $q \geq q_{0}$.

Proof of the claim: Suppose the map

$$
h_{X}(m): H^{1}\left(X, F^{n *} V(m)\right) \longrightarrow H^{1}\left(X, F^{n *} V(m+1)\right)
$$

is an isomorphism for some $m=m_{0} \geq 1$. Then we prove that $h^{1}\left(X, F^{n *} V(m)\right)=0$, for all $m \geq m_{0}$. Note that the map $h_{X}(m)$ is an isomorphism if and only if the canonical map

$$
H^{0}\left(X, F^{n *} V(m+1)\right) \longrightarrow H^{0}\left(X,\left.F^{n *} V(m+1)\right|_{Y}\right) \longrightarrow 0
$$

is surjective. We have the following commutative diagram of canonical maps, where the top horizontal map is surjective

$$
\begin{array}{ccc}
H^{0}\left(X, F^{n *} V\left(m_{0}+1\right)\right) \otimes H^{0}\left(X, \mathcal{O}_{X}(1)\right) & \longrightarrow & H^{0}\left(X,\left.F^{n *} V\left(m_{0}+1\right)\right|_{Y}\right) \otimes H^{0}\left(X, \mathcal{O}_{X}(1)\right) \\
H^{0}\left(X, F^{n *} V\left(m_{0}+2\right)\right) & \longrightarrow & H^{0}\left(X,\left.F^{n *} V\left(m_{0}+2\right)\right|_{Y}\right)
\end{array}
$$

Moreover the second vertical map is surjective as $Y$ is 0 -dimensional and $H^{0}\left(X, \mathcal{O}_{X}(1)\right)$ contains elements which do not vanish on $Y$. Hence the bottom horizontal map is surjective. By induction on $m$, it follows that the map

$$
H^{1}\left(X, F^{n *} V(m)\right) \longrightarrow H^{1}\left(X, F^{n *} V(m+1)\right)
$$

is an isomorphism, for all $m \geq m_{0}$. Since, by Serre vanishing $H^{1}\left(X, F^{n *} V(m)\right)=0$, for $m>>0$, we conclude that $h^{1}\left(X, F^{n *} V(m)\right)=0$, for all $m \geq m_{0}$.

Now let $q \geq q_{0}$. Then, we claim that the map $h_{X}(m)$ is an isomorphism for some $m \in\left[\left(x_{0}-d_{0}\right) q,\left(x_{0}-d_{0}\right) q+C_{1}\right):$ Otherwise

$h^{1}\left(X, F^{n *} V(m)\right)-h^{1}\left(X, F^{n *} V(m+1)\right) \geq 1, \forall m \in\left[\left(x_{0}-d_{0}\right) q,\left(x_{0}-d_{0}\right) q+C_{1}+1\right)$, which would imply that $h^{1}\left(X, F^{n *} V\left(\left\lceil\left(x_{0}-d_{0}\right) q\right)\right\rceil\right)>C_{1}$. But this contradicts the inequality (4.1). Hence the claim.

Therefore,

$$
\begin{gathered}
h^{1}\left(X, F^{n *} V(m)\right)=\ell\left(R / I^{[q]}\right)_{m+q d_{0}}=0, \quad \text { for all } m \geq\left(x_{0}-d_{0}\right) q+C_{1}+1 \text { and } q \geq q_{0} . \\
\Longrightarrow \mathbf{m}^{m+q d_{0}} \subset I^{[q]}, \quad \text { for all } m+q d_{0} \geq x_{0} q+C_{1}+1 \quad \text { and } q \geq q_{0} \\
\Longrightarrow c^{I}(\mathbf{m}) \leq \lim _{q \rightarrow \infty} \frac{x_{0} q+C_{1}+1}{q}=x_{0}=\alpha(R, I) .
\end{gathered}
$$


In higher dimensional case we get such an equality when the ring is strongly $F$ regular on the punctured spectrum or when Proj $R$ is smooth. We recall the following definition from [HH2].

Definition 4.7. A Noetherian domain $R$ such that $R \longrightarrow R^{1 / p}$ is module finite over $R$, is strongly $F$-regular if for every nonzero $c \in R$ there exists $q$ such that $R$-linear map $R \longrightarrow R^{1 / q}$ that sends 1 to $c^{1 / q}$ splits as a map of $R$-modules, i.e. iff $R c^{1 / q} \subseteq R^{1 / q}$ splits over $R$. A regular ring is strongly $F$-regular ([HH2]).

Since the following lemma is easy to check we state it without the proof.

Lemma 4.8. For a standard graded pair $(R, I)$ and for a fixed power $q_{0}$ of $p$

$$
f_{R, I}^{\left[q_{0}\right]}\left(q_{0} x\right)=q_{0}^{d-1} f_{R, I}(x), \text { for all } x \geq 0 .
$$

In particular $\alpha\left(R, I^{\left[q_{0}\right]}\right)=q_{0} \alpha(R, I)$.

Theorem 4.9. Let $(R, I)$ be a standard graded pair of dimension $d \geq 2$.

$$
R \text { is strongly F-regular on } \operatorname{Spec} R \backslash\{\mathbf{m}\} \Longrightarrow \alpha(R, I)=c^{I}(\mathbf{m}) \text {. }
$$

Thus the above equality holds for a standard graded pair $(R, I)$, provided $R$ is a domain and $X=$ Proj $R$ is strongly F-regular.

In particular the equality $\alpha(R, I)=c^{I}(\mathbf{m})$ holds for any two dimensional standard graded pair $(R, I)$, where $R$ is a normal domain (equivalently Proj $R$ is a nonsingular curve).

Proof. Is enough to prove that $\alpha(R, I) \geq c^{I}(\mathbf{m})$.

By Theorem 5.10 of [HH2], there exists $n_{0}$ such that $\mathbf{m}^{n_{0}} \subset \tau(R)$, where $\tau(R)$ denotes the test ideal of $R$. Hence, for every ideal $J$ of $R$ we have $\mathbf{m}^{n_{0}} J^{*} \subseteq J$ where $J^{*}$ denotes the tight closure of $J$ in $R$.

It is enough to show that, if $\beta \in \mathbb{N}[1 / p]$ with $\beta<c^{I}(\mathbf{m})$ then $\beta<\alpha(R, I)$. Let $2 \epsilon=c^{I}(\mathbf{m})-\beta>0$.

Now we choose a power $q_{0}$ of $p$ such that, for $q \geq q_{0}$, we have $\beta q \in \mathbb{N}, \epsilon q \geq n_{0}$ and $\mathbf{m}^{\beta q+\lfloor\epsilon q\rfloor} \nsubseteq I^{[q]}$. In particular $\mathbf{m}^{\beta q_{0}+n_{0}} \nsubseteq I^{\left[q_{0}\right]}$ and therefore $\mathbf{m}^{\beta q_{0}} \nsubseteq\left[I^{\left[q_{0}\right]}\right]^{*}$.

We choose a homogeneous element $z \in \mathbf{m}^{\beta q_{0}} \backslash\left[I^{\left[q_{0}\right]}\right]^{*}$. Let $J=\left(z, I^{\left[q_{0}\right]}\right)$. By [HH1], $e_{H K}\left(R, I^{\left[q_{0}\right]}\right)-e_{H K}(R, J)>0$ and $e_{H K}\left(R, I^{\left[q_{0}\right]}\right)=e_{H K}\left(R,\left[I^{\left[q_{0}\right]}\right]^{*}\right)$. Therefore, by Remark 2.15 of [T2],

$$
f_{R,\left[I^{\left.\left[q_{0}\right]\right]^{*}}\right.}(x)=f_{R, I^{\left[q_{0}\right]}}(x) \geq f_{R, J}(x), \quad \text { for all } x .
$$

Moreover, if $x<\beta q_{0}=\left\lfloor\beta q_{0}\right\rfloor$, then

$$
\operatorname{deg} z^{q} \geq q q_{0} \beta \Longrightarrow\left(R / I^{\left[q q_{0}\right]}\right)_{\lfloor x q\rfloor}=\left(R /\left(z^{q} R+I^{\left[q q_{0}\right]}\right)\right)_{\lfloor x q\rfloor}, \quad \text { for all } q .
$$

Hence $f_{R, I^{\left[q_{0}\right]}}(x)=f_{R, J}(x)$, for $x<\beta q_{0}$. In particular

$$
e_{H K}\left(R, I^{\left[q_{0}\right]}\right)-e_{H K}(R, J)=\int_{\beta q_{0}}^{\infty}\left(f_{R, I}^{\left[q_{0}\right]}(x)-f_{R, J}(x)\right) d x>0 .
$$

This implies $\alpha\left(R, I^{\left[q_{0}\right]}\right)>\beta q_{0}$ and by Lemma 4.8, $\alpha(R, I)>\beta$. Therefore $\alpha(R, I) \geq$ $c^{I}(\mathbf{m})$.

If Proj $R$ is strongly $F$-regular then for any nonzero homogeneous element $c \in \mathbf{m}$, the ring $R_{(c)}$ (the subring consisting of degree 0 elements of $R_{c}$ ) and therefore $R_{c}$ is strongly $F$-regular. Hence (by Theorem 5.10 of [HH2]) $\tau(R)$ is an $\mathbf{m}$-primary graded ideal. Now the above arguement proves the second assertion. 
The equality $\alpha(R, I)=c^{I}(\mathbf{m})$ also holds when $I$ is generated by a system of parameters and then (not surprisingly) the number is decided by the degrees of the generators.

Theorem 4.10. Let $R$ be a standard graded pair of dimension $d \geq 2$ over a perfect field of characteristic $p$ and $I$ be generated by homogeneous system of parameters $f_{1}, \ldots, f_{d}$ of degrees $n_{1}, n_{2}, \ldots, n_{d}$ respectively. Then

$$
\alpha(R, I)=c^{I}(\mathbf{m})=n_{1}+n_{2}+\cdots+n_{d} .
$$

Proof. By Lemma 3.2, we have $n_{1}+n_{2}+\cdots+n_{d}=\alpha(R, I)$. Enough to prove the following claim.

Claim. There exists a constant $l_{0} \in \mathbb{N}$ such that $\ell\left(R /\left(f_{1}^{q}, \ldots, f_{d}^{q}\right)\right)_{\lfloor x q\rfloor}=0$, for all $\lfloor x q\rfloor \geq\left(n_{1}+\cdots+n_{d}\right) q+l_{0}$.

Proof of the claim: We prove by induction on $d$. If $\operatorname{dim} R=1$ then we $k\left[f_{1}\right] \longrightarrow R$ is a finite graded map of degree 0 . Hence $R$ is a direct sum of free and torsion modules over the principal ideal domain $k\left[f_{1}\right]$. Therefore there exists $l_{0}$ such that $\ell\left(R /\left(f_{1}^{q}\right)\right)_{\lfloor x q\rfloor}=0$, for all $\lfloor x q\rfloor \geq n_{1} q+l_{0}$. Rest of the claim follows from the surjective map (3.1) from Section 2.

As a corollary we have bounds on $\alpha(R, I)$ in terms of the degrees of the generators of $I$. Moreover if $R$ is a polynomial ring then the bound is explicit.

Corollary 4.11. If $R$ is a standard graded $n$-dimensional ring and $I$ is generated by homogeneous elements $h_{1}, \ldots, h_{s}$ of degree $d_{1} \leq \cdots \leq d_{s}$ respectively. Then

(1) $d_{1} \leq \alpha(R, I) \leq c^{I}(\mathbf{m}) \leq d_{1}+\cdots+d_{s}$.

(2) If $R=k\left[X_{1}, \ldots, X_{n}\right]$ is a polynomial ring of dimension $\geq 2$ then

$$
\alpha(R, I)=c^{I}(\mathbf{m})=\max \left\{s \mid \mathbf{m}^{s} \nsubseteq I\right\}+n .
$$

Proof. (1) We choose an ideal $J$, which is generated by a system of parameters $\left\{h_{i_{1}}, \ldots, h_{i_{n}}\right\}$ $\subset\left\{h_{1}, \ldots, h_{s}\right\}$. Then, by Theorem 4.10 ,

$$
\alpha(R, I) \leq \alpha(R, J)=c^{J}(\mathbf{m})=d_{i_{1}}+\cdots+d_{i_{n}} \leq d_{1}+\cdots+d_{s} .
$$

(2) The second assertion follows from Theorem 4.9 and [HMTW], Example 2.7 (iii).

The equality $\alpha(R, I)=c^{I}(\mathbf{m})$ carries over to the Segre product of standard graded pairs.

Lemma 4.12. Let $\left(R_{1}, I_{1}\right), \ldots,\left(R_{r}, I_{r}\right)$ be standard graded pairs of dimension $\geq 2$, with their respective graded maximal ideals $\mathbf{m}_{1}, \ldots, \mathbf{m}_{r}$ such that $c^{I_{i}}\left(\mathbf{m}_{i}\right)=\alpha\left(R_{i}, I_{i}\right)$, for every $i$. Then

$$
c^{I_{1} \# \cdots \# I_{r}}\left(\mathbf{m}_{1} \# \cdots \# \mathbf{m}_{r}\right)=\alpha\left(R_{1} \# \cdots \# R_{r}, I_{1} \# \cdots \# I_{r}\right)
$$

and therefore

$$
c^{I_{1} \# \cdots \# I_{r}}\left(\mathbf{m}_{1} \# \cdots \# \mathbf{m}_{r}\right)=\max \left\{c^{I_{i}}\left(\mathbf{m}_{i}\right) \mid 1 \leq i \leq r\right\} .
$$

Proof. Let $(R, I)$ and $(S, J)$ be two standard graded pairs of dimensions $d_{1}$ and $d_{2}$, respectively, over a field $k$ of characteristic $p>0$. Then, by Proposition 2.17 of [T2]

$$
f_{R \# S, I \# J}=\left(F_{R}\right)\left(f_{S, J}\right)+\left(F_{S}\right)\left(f_{R, I}\right)-\left(f_{R, I}\right)\left(f_{S, J}\right),
$$

where $F_{R}(x)=e(R) x^{d_{1}-1} /\left(d_{1}-1\right)$ ! and $F_{S}(x)=e(S) x^{d_{2}-1} /\left(d_{2}-1\right)$ !.

Claim. $\quad \alpha(R \# S, I \# J)=\max \{\alpha(R, I), \alpha(S, J)\}$. 
Proof of the claim: Let $\alpha_{1}=\alpha(R, I)$ and $\alpha_{2}=\alpha(S, J)$. Without loss of generality, one can assume that $\alpha_{1} \leq \alpha_{2}$. It is enough to show $\alpha(R \# S, I \# J) \geq \alpha_{2}$. For any $\epsilon_{1}>0$, there exists $0<\epsilon<\epsilon_{1}$ such that for $x \in\left[\alpha_{2}-\epsilon, \alpha_{2}\right), f_{S, J}(x) \neq 0$ and hence

$$
f_{R \# S, I \# J}(x)=f_{R, I}(x)\left(F_{S}(x)-f_{S, J}(x)\right)+F_{R}(x) f_{S, J}(x)>0 .
$$

Moreover, it follows from the definition that $c^{I \# J}(\mathbf{m} \# \mathbf{n}) \leq \max \left\{c^{I}(\mathbf{m}), c^{J}(\mathbf{n})\right\}$. Hence, for $\left(R_{i}, \mathbf{m}_{i}\right)$ and $I_{i}$ as given above, we have

$$
\begin{aligned}
c^{I_{1} \# \cdots \# I_{r}}\left(\mathbf{m}_{1} \# \cdots \# \mathbf{m}_{r}\right) & \leq \max \left\{c^{I_{i}}\left(\mathbf{m}_{i}\right) \mid 1 \leq i \leq r\right\} \\
=\max \left\{\alpha\left(R_{i}, I_{i}\right) \mid 1 \leq i \leq r\right\} & =\alpha\left(R_{1} \# \cdots \# R_{r}, I_{1} \# \cdots I_{r}\right) .
\end{aligned}
$$

On the other hand, by Proposition 4.4, we have

$$
\alpha\left(R_{1} \# \cdots \# R_{r}, I_{1} \# \cdots \# I_{r}\right) \leq c^{I_{1} \# \cdots \# I_{r}}\left(\mathbf{m}_{1} \# \cdots \# \mathbf{m}_{r}\right) .
$$

This proves the lemma.

We can summarize the results of this subsection in Theorem E (stated in the introduction)

Proof of Theorem E. It is a consequence of Proposition 4.4, Proposition 4.6, Theorem 4.9, Theorem 4.10] and Lemma 4.12, $\square$

\subsection{The shape of the graph of $f_{R, \mathbf{m}}$ and regularity.}

Theorem 4.13. Let $R$ be a standard graded ring of dimension $d \geq 2$ defined over a perfect field of characteristic $p>0$.

Then $\alpha(R, \mathbf{m}) \leq d$. Moreover, the equality holds if and only if there exists a prime ideal $P$, such that $\operatorname{dim} R / P=d$ and $R / P$ is regular.

In particular, if $R$ is a domain then $\alpha(R, \mathbf{m})=d$ if and only if $R$ is regular.

Proof. (1) Let $J$ be a parameter ideal generated by elements of degree 1. By Lemma 3.2 , $\alpha(R, J)=d$. Hence $\alpha(R, \mathbf{m}) \leq \alpha(R, J) \leq d$.

(2) Suppose $R / P$ is regular for some $P \in \operatorname{Assh}(R)$. Then, by Corollary 3.3, we have $\alpha(R / P, \mathbf{m} / P)=d$. Now the additivity of the HK density function implies that $d \geq \alpha(R, \mathbf{m})=\max \{\alpha(R / P, \mathbf{m} / P) \mid P \in \operatorname{Assh}(R)\} \geq d$.

Conversely, suppose $\alpha(R, \mathbf{m})=d$. Let $P \in \operatorname{Assh}(R)$ such that $\alpha(R / P, \mathbf{m} / P)=d$. Suppose $R / P$ is not regular. We choose a system of parameter ideal $J=\left(x_{1}, \ldots, x_{d}\right)$ of linear forms such that $\left\{x_{1}, \ldots, x_{d}\right\}$ is a part of a minimal set of generators of $\mathbf{m} / P$. Then

$$
\left(J^{*}\right) \cap(R / P)_{1}=J \cap(R / P)_{1} \neq(\mathbf{m} / P) \cap(R / P)_{1},
$$

where the first equality follows from Theorem 2.2 of $[\mathrm{S}]$.

Hence, by Corollary 3.2 of [HMTW], we have $c^{\mathbf{m} / P}(J)<d$. On the other hand, by Proposition 1.7 of $[\mathrm{MTW}], c^{\mathbf{m} / P}(J)=c^{\mathbf{m} / P}(\mathbf{m} / P)$, as $\mathbf{m} / P=$ the integral closure of $J$ in $R / P$.

But then $\alpha(R / P, \mathbf{m} / P) \leq c^{\mathbf{m} / P}(\mathbf{m} / P)<d$, which is a contradiction.

(3) Assertion (3) is a particular case of Assertion (2).

Theorem 4.14. Let $R$ be a standard graded domain of dimension $d \geq 2$. Then the function $f_{R, \mathbf{m}}$ is symmetric at $x=d / 2$, i.e.,

$$
f_{R, \mathbf{m}}(x)=f_{R, \mathbf{m}}(d-x), \quad \text { for all } x \text { iff } R \text { is a regular ring. }
$$

Moreover, if $\operatorname{dim} R=2$ then either

(1) $f_{R, \mathbf{m}}$ is symmetric at $x=1$, or 
(2) $f_{R, \mathbf{m}}(1-y)>f_{R, \mathbf{m}}(1+y)$, for all $y \in(0,1)$.

Proof. If $R$ is regular then $\mathbf{m}$ is generated by a system of parameters of degree 1 and therefore, by Corollary 3.3, the function $f_{R, \mathbf{m}}$ is symmetric at $d / 2$.

Conversely, if $f_{R, \mathbf{m}}$ is symmetric at $d / 2$ then $\alpha(R, \mathbf{m})=d$, hence, by Lemma 4.13 , the ring $R$ is regular.

We give the proof of second assertion in the end of subsection 6.2

In Section 6 we study, in more detail, the invariant $\alpha(R, I)$ for two dimensional domains. Here we also explicitly express the HK density function $f_{R, I}$ in terms of the strong Harder-Narasimhan slopes of the associated syzygy bundles. We recall some well known relevant results about vector bundles.

\section{Some Basic facts about Semistability properties of VeCtor BundLes}

Let $X$ be a nonsingular projective curve over an algebraically closed field $k$.

Definition 5.1. A vector bundle $V$ on $X$ is semistable if for every proper subbundle $W \subset V$ we have $\mu(W) \leq \mu(V)$, where $\mu(V)=\operatorname{deg}(V) / \operatorname{rank}(V)$.

The bundle $V$ is strongly semistable (provided char $k=p>0$ ) if, for every $m^{\text {th }}$ iterated Frobenius map $F^{m}: X \longrightarrow X$, the bundle $F^{m *}(V)$ is semistable, which is equivalent to the statement that $\pi^{*} V$ is semistable for every finite map $\pi: Y \longrightarrow X$ (because the pull back of a semistable bundle is semistable for any finite separable map).

Note that when char $k=0$, then $V$ semistable implies $\pi^{*} V$ is semistable for every finite map $\pi: Y \longrightarrow X$ (see [HL]). Hence the notions of strongly semistability and semistability coincide in char $k=0$.

Definition 5.2. Every vector bundle $V$ on $X$ has the unique filtration of subbundles

$$
0=F_{0} \subset F_{1} \subset \cdots \subset F_{t} \subset F_{t+1}=V,
$$

called the Harder-Narasimhan (HN) filtration of $V$, satisfying the following conditions

(1) for every $i$, the bundle $F_{i} / F_{i-1}$ is semistable and

(2) $\mu\left(F_{1}\right)>\mu\left(F_{2} / F_{1}\right)>\ldots>\mu\left(F_{t+1} / F_{t}\right)\left(\equiv \mu\left(F_{1}\right)>\mu\left(F_{2}\right)>\ldots>\mu(V)\right)$.

If in addition the bundles $F_{i} / F_{i-1}$ is strongly semistable, for every $i$ then we call the filtration (5.1) a strong Harder-Narasimhan filtration (or a strong HN filtration).

Notations 5.3. Let $V$ be a vector bundle on a nonsingular projective curve $X$.

(1) If $V$ has the HN filtration as in (5.1) then we define $\mu_{i}=\mu\left(F_{i} / F_{i-1}\right)$, the $H N$ slopes of $V$ and $r_{i}=\operatorname{rank}\left(F_{i} / F_{i-1}\right)$, the HN ranks of $V$. We denote the minimum HN slope $\mu_{\min }(V)=\mu\left(V / F_{t}\right)$. We call the set

$$
\left(\left\{\mu_{1}, \mu_{2}, \cdots, \mu_{t+1}\right\},\left\{r_{1}, \ldots, r_{t+1}\right\}\right)
$$

the $H N$ data for $V$.

(2) By Theorem 2.7 of [L], if char $k=p>0$, then for a given bundle $V$, there exists $m>0$ such that the HN filtration of $F^{m *} V$ is the strong HN filtration

$$
0=E_{0} \subset E_{1} \subset \cdots \subset E_{l} \subset E_{l+1}=F^{m *} V .
$$

In this case we define $a_{i}=\left(1 / p^{m}\right) \mu\left(E_{i} / E_{i-1}\right)$ the strong $H N$ slopes of $V$. We denote the minimum strong $H N$ slope and the strong $H N$ data of $V$ by

$$
a_{\min }(V)=\left(1 / p^{m}\right) \mu\left(E_{l+1} / E_{l}\right) \quad \text { and } \quad\left(\left\{a_{1}, \ldots, a_{l+1}\right\},\left\{\tilde{r}_{1}, \ldots, \tilde{r}_{l+1}\right\}\right),
$$


respectively, where $\tilde{r}_{i}=\operatorname{rank}\left(E_{i+1} / E_{i}\right)$.

The notion of the strong HN slopes and the strong HN data are well defined because if $V$ has a strong HN filtration, then for every $n \geq 1$, the $n^{\text {th }}$ Frobenius pull back of the HN filtration of $V$ is the strong $\mathrm{HN}$ filtration for $F^{n *} V$.

Remark 5.4. Let $\mathcal{O}_{X}(1)$ be an ample line bundle of degree $d$ on $X$. Let $\tilde{E}$ be a semistable vector-bundle on $X$ with $\mu(\tilde{E})=\mu$ and $\operatorname{rank}(\tilde{E})=r$. Then by Serre duality

$$
\begin{array}{lll}
m<-\mu / d & \Longrightarrow & h^{1}(X, \tilde{E}(m))=-r(\mu+d m+(g-1)) \\
-\mu / d \leq m \leq-\mu / d+(d-3) & \Longrightarrow & h^{1}(X, \tilde{E}(m))=C \\
-\mu / d+(d-3)<m & \Longrightarrow & h^{1}(X, \tilde{E}(m))=0,
\end{array}
$$

where $|C| \leq r(g-1)$ and $g=\operatorname{genus}(X)$.

Remark 5.5. Let $V$ be a vector bundle on $X$ with the HN filtration (5.1).

(1) If $V$ has a nontrivial $\mathrm{HN}$ filtration (i.e. $V$ is not semistable) then $\mu_{\min }(V)<$ $\mu(V)$.

(2) If $0 \longrightarrow V^{\prime} \longrightarrow V \longrightarrow V^{\prime \prime} \longrightarrow 0$ is a short exact sequence of nonzero vector bundles on $X$, then

(a) either $\mu\left(V^{\prime}\right) \leq \mu(V) \leq \mu\left(V^{\prime \prime}\right)$ or $\mu\left(V^{\prime}\right) \geq \mu(V) \geq \mu\left(V^{\prime \prime}\right)$.

(b) For a nonzero map of bundles $E \longrightarrow W$, where $W$ is semistable, $\mu_{\min }(E) \leq$ $\mu(W)$ : We choose a semistable subquotient bundle $E_{1}$ (occuring in the HN filtration of $E$ ) such that the restricted map $E_{1} \longrightarrow W$ is nonzero. This gives $\mu_{\text {min }}(E) \leq \mu\left(E_{1}\right) \leq \mu\left(\operatorname{Im}\left(E_{1}\right)\right) \leq \mu(W)$.

(c) $a_{\min }(V) \leq \mu_{\min }(V)$ : Enough to prove $\mu_{\min }\left(F^{m *}(V)\right) \leq p^{m} \mu_{\min }(V)$, for any $m \geq 1$. Let $V_{1}$ be the semistable quotient bundle of $V$ such that $\mu_{\min }(V)=\mu\left(V_{1}\right)$. Let $W$ be the semistable quotient bundle of $F^{m *}\left(V_{1}\right)$ such that $\mu_{\min }\left(F^{m *} V_{1}\right)=\mu(W)$. Now the nonzero map $F^{m *} V \longrightarrow W$ gives

$$
\mu_{\text {min }}\left(F^{m *} V\right) \leq \mu(W) \leq \mu\left(F^{m *}\left(V_{1}\right)\right)=p^{m} \mu\left(V_{1}\right)=p^{m} \mu_{\min }(V) .
$$

\section{The HK DENSITY FUnCTIONS FOR TWO DIMENSIONAL RINGS}

6.1. The HK density functions for vector bundles on curves. Let $X$ be a nonsingular projective curve over an algebraically closed field of characteristic $p>0$. Let $\mathcal{O}_{X}(1)$ be an ample line bundle of degree $d$ on $X$. Let $V$ be a vector bundle on $X$. For the notion of HN data, (strong) HN slopes and the minimum strong HN slope $a_{\min }(V)$, for a vector bundle $V$ on $X$, we refer to Notations 5.3 .

We define the HK density function of $V$ (a continuous but not necessarily compactly supported function) with respect to $\mathcal{O}_{X}(1)$ (where $q=p^{n}$ )

$$
f_{V, \mathcal{O}_{X}(1)}: \mathbb{R} \longrightarrow[0, \infty) \text { given by } x \rightarrow \lim _{n \rightarrow \infty} \frac{1}{q} h^{1}\left(X, F^{n *} V(\lfloor(x-1) q\rfloor)\right) .
$$

This function is well defined and is given explicitly as follows. Let the strong HN data for $V$ be given by $\left(\left\{a_{1}, \ldots, a_{l+1}\right\},\left\{r_{1}, \ldots, r_{l+1}\right\}\right)$. Then there is $m_{1}$ such that $F^{m_{1} *} V$ has the strong HN filtration

$$
0=E_{0} \subset E_{1} \subset \cdots \subset E_{l} \subset E_{l+1}=F^{m_{1} *} V,
$$

where $a_{i}=\left(1 / p^{m_{1}}\right) \mu\left(E_{i} / E_{i-1}\right)$ and $r_{i}=\operatorname{rank}\left(E_{i} / E_{i-1}\right)$.

Since $a_{1}>a_{2}>\cdots>a_{l+1}$, we can choose $q>>0$ such that

$$
-\frac{a_{1} q}{d}<-\frac{a_{1} q}{d}+(d-3)<-\frac{a_{2} q}{d}<-\frac{a_{2} q}{d}+(d-3)<\cdots<-\frac{a_{l+1} q}{d} .
$$


By Remark 5.4

$$
h^{1}\left(X, F^{n+m_{1} *} V(\lfloor(x-1) q\rfloor)\right)=\sum_{i=1}^{l+1} h^{1}\left(X, F^{n *}\left(E_{i} / E_{i-1}\right)(\lfloor(x-1) q\rfloor)\right),
$$

where $q=p^{n}$. Taking limit as $n \rightarrow \infty$, we get

$$
\begin{array}{lll}
x<1-a_{1} / d & \Longrightarrow & f_{V, \mathcal{O}_{X}(1)}(x)=-\left[\sum_{i=1}^{l+1} a_{i} r_{i}+d(x-1) r_{i}\right] \\
1-a_{i} / d \leq x<1-a_{i+1} / d & \Longrightarrow & f_{V, \mathcal{O}_{X}(1)}(x)=-\left[\sum_{k=i+1}^{l+1} a_{k} r_{k}+d(x-1) r_{k}\right] \\
1-a_{l} / d \leq x<1-a_{l+1} / d & \Longrightarrow & f_{V, \mathcal{O}_{X}(1)}(x)=-\left[a_{l+1} r_{l+1}+d(x-1) r_{l+1}\right] \\
1-a_{l+1} / d \leq x & \Longrightarrow & f_{V, \mathcal{O}_{X}(1)}(x)=0 .
\end{array}
$$

This implies Support $f_{V, \mathcal{O}_{X}(1)} \subseteq\left(-\infty, 1-a_{\min }(V) / d\right]$ and

$$
\alpha\left(V, \mathcal{O}_{X}(1)\right):=\operatorname{Sup}\left\{x \mid f_{V, \mathcal{O}_{X}(1)}(x)>0\right\}=1-\frac{a_{\min }(V)}{d} .
$$

Remark 6.1. Replacing $R$ by $R \otimes_{k} \bar{k}$ does not change the function $f_{R, I}$ and the semistability behaviour of any vector bundle $V$ on $X=\operatorname{Proj} R$. Therefore we can assume, without loss of generality, that the underlying field $k$ is algebraically closed.

\subsection{The HK density functions of $(R, I)$ and its syzygy bundles.}

Notations 6.2. Let $(R, I)$ be a standard graded pair, where $R$ is a domain defined over a perfect field. Let $h_{1}, \ldots, h_{\mu}$ be a set of generators of $I$ with deg $h_{i}=d_{i}$.

Let $S=\oplus_{m \geq 0} S_{m}$ be the integral closure of $R$ in its quotient field. Since $R$ is a standard graded ring over $k$, the canonical embedding $Y=\operatorname{Proj} R \longrightarrow \mathbb{P}_{k}^{n}$ gives the very ample line bundle $\mathcal{O}_{Y}(1)$ on $Y$. Let $X=\operatorname{Proj} S$ and let $\mathcal{O}_{X}(1)$ be the pull back of $\mathcal{O}_{Y}(1)$ under the map $X \longrightarrow Y$. Let

$$
0 \longrightarrow V \longrightarrow M=\oplus_{i=1}^{\mu} \mathcal{O}_{X}\left(1-d_{i}\right) \longrightarrow \mathcal{O}_{X}(1) \longrightarrow 0,
$$

be the canonical (locally split) exact sequence of locally free sheaves of $\mathcal{O}_{X}$-modules, where the map $\mathcal{O}_{X}\left(1-d_{i}\right) \longrightarrow \mathcal{O}_{X}(1)$ is given by the multiplication by the the element $h_{i}$.

Note that, in the case $I$ generated by degree 1 elements, the HK density function (and hence the maximum support of $f_{R, I}$ ) has been explicitly given in [T2].

Theorem 6.3. With the above notations, we have

(1) $f_{R, I}(x)=f_{V, \mathcal{O}_{X}(1)}(x)-f_{M, \mathcal{O}_{X}(1)}(x)$, for $x \geq 0$ and

(2) $\alpha(R, I)=1-a_{i}(V) / d$ or $1-a_{i}(M) / d$, where $a_{i}(V)\left(a_{i}(M)\right)$ is one of the strong $H N$ slopes of $V$ (respectively $M$ ) and hence $\alpha(R, I)$ is a rational number. Moreover

(3) if $d_{1}=\cdots=d_{\mu}$ then $\alpha(R, I)=1-a_{\min }(V) / d$.

Proof. We note that the inclusion map $\pi: R \longrightarrow S$ is a graded finite map of degree 0 , where $S$ is a normal domain and $Q(R)=Q(S)$. The additivity of the HK density function implies that

$$
f_{R, I}(x)=f_{S, I}(x)=\lim _{n \rightarrow \infty} f_{n}(S, I)(x)=\lim _{n \rightarrow \infty} \frac{1}{q} \ell\left(\frac{S}{I^{[q] S}}\right)_{\lfloor x q\rfloor} .
$$

Also $X$ is a nonsingular projective curve. 
Let $m_{0}>0$ be such that, for $m \geq m_{0}$, we have $H^{1}\left(X, \mathcal{O}_{X}(m)\right)=0$ and $H^{0}\left(X, \mathcal{O}_{X}(m)\right)=$ $S_{m}$. Then, for $m \geq m_{0}, n \geq 0$ and $q=p^{n}$, the long exact sequence of cohomologies

$$
\begin{gathered}
0 \longrightarrow H^{0}\left(X,\left(F^{n *} V\right)(m)\right) \longrightarrow H^{0}\left(X,\left(F^{n *} M\right)(m)\right) \stackrel{\varphi_{m, q}}{\longrightarrow} H^{0}\left(X, \mathcal{O}_{X}(q+m)\right) \\
\longrightarrow H^{1}\left(X,\left(F^{n *} V\right)(m)\right) \longrightarrow H^{1}\left(X,\left(F^{n *} M\right)(m)\right) \longrightarrow 0
\end{gathered}
$$

gives

$$
f_{n}\left(\frac{m+q}{q}\right)=\frac{1}{q} \ell\left(\frac{S}{I[q] S}\right)_{m+q}=\frac{1}{q}\left[h^{1}\left(X,\left(F^{n *} V\right)(m)\right)-h^{1}\left(X,\left(F^{n *} M\right)(m)\right)\right] .
$$

Hence $f_{R, I}(x)=f_{V, \mathcal{O}_{X}(1)}(x)-f_{M, \mathcal{O}_{X}(1)}(x)$, which proves the assertion (1).

The second assertion follows from the description of the HK density function of a vector bundle in terms of its strong $\mathrm{HN}$ data.

If $d_{1}=\ldots=d_{\mu}$ then $M$ is a strongly semistable bundle (being a sum of line bundles of the same degrees). Now, by Remark $5.5($ as $\mu(M)<\mu(\mathcal{L}))$

$$
a_{\min }(V) \leq \mu_{\min }(V) \leq \mu(V)<\mu(M)=a_{\min }(M) .
$$

Therefore $\alpha(R, I)=\alpha\left(V, \mathcal{O}_{X}(1)\right)=1-a_{\min }(V) / d$.

Now we are ready to give a proof of

Theorem 4.14 (2) If $R$ is a standard graded domain of dimension 2 then either

(1) $f_{R, \mathbf{m}}$ is symmetric at $x=1$, or

(2) $f_{R, \mathbf{m}}(1-y)>f_{R, \mathbf{m}}(1+y)$, for all $y \in(0,1)$.

Proof. If $R$ is a regular ring then by Assertion (1), the function $f_{R, \mathbf{m}}$ is symmetric at $x=1$.

Let $R$ be a nonregular domain of dimension 2 .

We consider the sequence (6.3)

$$
0 \longrightarrow V \longrightarrow M=\oplus^{s} \mathcal{O}_{X} \longrightarrow \mathcal{O}_{X}(1) \longrightarrow 0,
$$

for the pair $(R, \mathbf{m})$, where $h_{1}, \ldots, h_{s}$ is a minimal set of degree 1 generators of $\mathbf{m}$.

Since $R$ is not regular, $s \geq \mu(\mathbf{m}) \geq 3$ and therefore rank $V=r=s-1 \geq 2$. If $\left(\left\{a_{1}>\ldots>a_{l+1}\right\},\left\{r_{1}, \ldots, r_{l+1}\right\}\right)$ denotes the strong HN data for $V$ then $l+1 \geq 1$. Also the strong HN data for $M$ is $(\{\mu(M)=0\},\{\operatorname{rank}(M)=s\})$. Hence

$$
y \in\left[0,-a_{1} / d\right) \Longrightarrow f_{R, \mathbf{m}}(1+y)=d-r d y<d-d y=f_{R, \mathbf{m}}(1-y) .
$$

Suppose there is $y_{0} \in(0,1)$ such that $f_{R, \mathbf{m}}\left(1-y_{0}\right) \leq f_{R, \mathbf{m}}\left(1+y_{0}\right)$.

Then $-a_{j} / d \leq y_{0}<-a_{j+1} / d$, for some $1 \leq j \leq l$. Hence

$$
-\sum_{i \geq j+1}\left(a_{i} r_{i}-d y_{0} r_{i}\right) \geq d-d y_{0}
$$

This gives

$$
d y_{0}\left(1-\sum_{i \geq j+1} r_{i}\right) \geq d+\sum_{i \geq j+1} a_{i} r_{i}=-\sum_{i=1}^{l+1} a_{i} r_{i}+\sum_{i \geq j+1} a_{i} r_{i}=-\sum_{i=1}^{j} a_{i} r_{i} .
$$

Since $j+1 \leq l+1$ and $-a_{i} \geq 0$, for $i$, implies that both the sides of the above equation are $=0$. In particular $j=l=1$ and $r_{l+1}=1=r_{2}$ and $a_{1}=0$. Hence, if for some $m_{1}, F^{m_{1} *}(V)$ has the strong $\mathrm{HN}$ filtration then it is given by

$$
0=E_{0} \subset E_{1} \subset E_{2}=F^{m_{1} *} V,
$$


where rank $E_{1}=\operatorname{rank} V-r_{2}=\mu(\mathbf{m})-2 \geq 1$ and deg $E_{1}=a_{1}=0$. Since $E_{1} \subseteq$ $\oplus^{s} \mathcal{O}_{X}$, we deduce that $E_{1}$ is trivial vector bundle of rank $\geq 1$. Hence $h^{0}\left(X, F^{m_{1} *} V\right) \geq$ $h^{0}\left(X, E_{1}\right) \geq 1$.

On the other hand, the linear independence of the $\left\{h_{1}, \ldots, h_{s}\right\} \in h^{0}\left(X, \mathcal{O}_{X}(1)\right)$ over the field $k$ implies that the set $\left\{h_{1}^{q}, \ldots, h_{s}^{q}\right\} \in H^{0}\left(X, \mathcal{O}_{X}(q)\right)$ is a linearly independent set over $k$. Therefore the map (as in (6.4))

$$
\varphi_{0, q}: \oplus^{s} H^{0}\left(X, \mathcal{O}_{X}\right) \longrightarrow H^{0}\left(X, \mathcal{O}_{X}(q)\right)
$$

is injective. But then $h^{0}\left(X, F^{m_{1} *} V\right)=0$, which is a contradiction. Hence we conclude that $f_{R, \mathbf{m}}(1+y)<f_{R, \mathbf{m}}(1-y)$, for every $y \in(0,1)$.

6.3. The $F$-threshold $c^{I}(\mathbf{m})$ and $\alpha(R, I)$ in characteristic 0 . We recall a well known notion of spread. We restrict our attention to the relevant situation.

Definition 6.4. Let $X$ be a nonsingular projective curve over an algebraically field of char 0 and let $V$ be a vector bundle on $X$. Then the triple $\left(A, X_{A}, V_{A}\right)$ is a spread for the pair $(X, V)$, if $A \subset k$ is a finitely generated $\mathbb{Z}$-algebra and $X_{A}$ is a projective scheme over $A$, and $V_{A}$ is a coherent, locally free sheaf over $X_{A}$ with

$$
X_{A} \otimes_{A} k=X \text { and } V_{A} \otimes_{A} k=V .
$$

By [EGA IV], we can further choose $A$ (if necessary, replacing $A$ by a finitely generated $\mathbb{Z}$-algebra $A_{0}$ such that $A \subseteq A_{0} \subset k$ ) so that $X_{A}$ is a smooth projective $A$-scheme. Moreover if

$$
0 \subset E_{1} \subset \cdots \subset E_{l} \subset V
$$

is the HN filtration of $V$ then $\left\{E_{i A}\right\}_{i}$ are coherent, locally free sheaves with a filtration $0 \subset E_{1 A} \subset \cdots \subset E_{l A} \subset V_{A}$ such that for all closed points $s \in \operatorname{Spec} A$,

$$
0 \subset E_{1(s)} \subset \cdots \subset E_{l(s)} \subset V_{s}
$$

is the $\mathrm{HN}$ filtration of $V_{s}:=V_{A} \otimes_{A} \overline{k(s)}$ as sheaves over $X_{s}:=X_{A} \otimes_{A} \overline{k(s)}$, where $E_{i s}:=E_{i A} \otimes_{A} \overline{k(s)}$ (this follows by the openness property of semistable vector bundles proved in $[\mathrm{Ma}])$.

Similarly for a pair $(R, I)$, where $R$ is a finitely generated $\mathbb{N}$ (standard) graded two dimensional domain over a field of characteristic 0 and $I \subset R$ is a homogeneous ideal of finite colength, then the triple $\left(A, R_{A}, I_{A}\right)$ is a spread of $(R, I)$, if $A$ is a finitely generated $\mathbb{Z}$-algebra $A \subset k$ and $R_{A}$ is a finitely generated $\mathbb{N}$ (standard) graded algebra over $A$ and $I_{A} \subset R_{A}$ is a homogeneous ideal such that

$$
R_{A} \otimes_{A} k=R \quad \text { and } \operatorname{Im}\left(I_{A} \otimes_{A} k\right)(\subset R)=I .
$$

We can choose such an $A$ so that for any closed point $s \in \operatorname{Spec} A$ the $\operatorname{ring} R_{s}=$ $R_{A} \otimes_{A} \overline{k(s)}$ is a finitely generated $\mathbb{N}$ (standard) graded 2-dimensional domain (which is a normal domain if $R$ is normal) over $\overline{k(s)}$ and the ideal $I_{s}=\operatorname{Im}\left(I_{A} \otimes_{A} \overline{k(s)}\right) \subset R_{s}$ is a homogeneous ideal of finite colength.

Moreover, if $\left(A, X_{A}, V_{A}\right)$ is a spread for $(X, V)$ and $\left(A, R_{A}, I_{A}\right)$ is a spread for $(R, I)$ then for any finitely generated $\mathbb{Z}$-algebra $A^{\prime}$ with $A \subset A^{\prime} \subset k$, the triple $\left(A^{\prime}, X_{A^{\prime}}, V_{A^{\prime}}\right)$ $\left(\left(A^{\prime}, R_{A^{\prime}}, I_{A^{\prime}}\right)\right)$ also satisfies the same properties as $\left(A, X_{A}, V_{A}\right)\left(\left(A, R_{A}, I_{A}\right)\right.$ respectively). Hence we may always assume that the spread (as above) is chosen such that $A$ contains a given finitely generated $\mathbb{Z}$-algebra $A_{0} \subseteq k$. 
Notations 6.5. Let $R$ be a standard graded domain over a field $k$ of characteristic 0 with a homogeneous ideal $I$ of finite colength such that $I$ is generated by the same degree elements. Let $S$ be the integral closure. Also let $X=$ Proj $S$ be the nonsingular projective curve over $k$ with a vector bundle $V$ given by the canonical exact sequence of the sheaves of $\mathcal{O}_{X}$-modules

$$
0 \longrightarrow V \longrightarrow \oplus^{\mu} \mathcal{O}_{X}\left(1-d_{0}\right) \longrightarrow \mathcal{O}_{X}(1) \longrightarrow 0,
$$

where $I$ is generated by $\mu$ homogeneous generators of degree $d_{0}$ each.

For $(X, V),(R, I)$ and $(S, I S)$, we can, respectivey, choose spreads $\left(A, X_{A}, V_{A}\right)$, $\left(A, R_{A}, I_{A}\right),\left(A, S_{A}, I S_{A}\right)$, as above, such that, in addition, for every $s \in \operatorname{Spec} A, S_{s}$ is the intergral closure of $R_{s}$ and $V_{s}$ is the syzygy bundle given by the canonical short exact sequence of locally free sheaves of $\mathcal{O}_{X_{s}}$-modules

$$
0 \longrightarrow V_{s} \longrightarrow \oplus^{\mu} \mathcal{O}_{X_{s}}\left(1-d_{0}\right) \longrightarrow \mathcal{O}_{X_{s}}(1) \longrightarrow 0 .
$$

Remark 6.6. If $\tilde{E}=V$ or $\tilde{E}=\oplus^{\mu} \mathcal{O}_{X_{s}}\left(1-d_{0}\right)$ is a vector bundle on $X$ and $\left(A, X_{A}, \tilde{E}_{A}\right)$ is a spread for $(X, \tilde{E})$ (as given above). Then (see the proof of Theorem 4.6 in [T4])

$$
f_{\tilde{E}, \mathcal{O}_{X}(1)}^{\infty}:=\lim _{p_{s} \rightarrow \infty} f_{\tilde{E}_{s}, \mathcal{O}_{X_{s}}(1)} \text { exists, }
$$

where $s \in \operatorname{Spec}(A)$ are closed points and $p_{s}=$ characteristic of $R_{s}$. Moreover, if

$$
0=E_{0} \subset E_{1} \subset \cdots \subset E_{l} \subset E_{l+1}=\tilde{E}
$$

is the HN filtration of $\tilde{E}$ such that $\mu_{i}=\mu\left(E_{i} / E_{i-1}\right)$ and $r_{i}=\operatorname{rank}\left(E_{i} / E_{i-1}\right)$ then

$$
\begin{aligned}
& 1 \leq x<1-\mu_{1} / d \quad \Longrightarrow \quad f_{\tilde{E}, \mathcal{O}_{X}(1)}^{\infty}(x)=-\left[\sum_{i \geq 1} \mu_{i} r_{i}+d(x-1) r_{i}\right] \\
& 1-\mu_{i} / d \leq x<1-\mu_{i+1} / d \Longrightarrow f_{\tilde{E}, \mathcal{O}_{X}(1)}^{\infty}(x)=-\left[\sum_{k \geq i+1} \mu_{k} r_{k}+d(x-1) r_{k}\right] \text {. }
\end{aligned}
$$

Theorem 6.7. Let $(R, I)$ be a standard graded pair and $\left(A, R_{A}, I_{A}\right)$ and $\left(A, X_{A}, V_{A}\right)$ be associated spreads as in Notations 6.5. Let $s$ denote a closed point of $\operatorname{Spec}(A)$ and $p_{s}=$ char $R_{s}$. Then

(1) for every $x \geq 0, f_{R, I}^{\infty}(x):=\lim _{p_{s} \rightarrow \infty} f_{R_{s}, I_{s}}(x)$ exists and the function $f_{R, I}^{\infty}:[0, \infty) \longrightarrow[0, \infty)$ is a continuous compactly supported function with

$$
\alpha^{\infty}(R, I):=\operatorname{Sup}\left\{x \mid f_{R, I}^{\infty}(x) \neq 0\right\}=1-\frac{\mu_{\min }(V)}{d} .
$$

(2) $\lim _{p_{s} \rightarrow \infty} \alpha\left(R_{s}, I_{s}\right)=\alpha^{\infty}(R, I)$.

(3) $\alpha\left(R_{s}, I_{s}\right) \geq \alpha^{\infty}(R, I)$, for $p_{s}>>0$. If, in addition, the bundle $V$ is semistable then

$$
\alpha\left(R_{s}, I_{s}\right)=\alpha^{\infty}(R, I) \Longleftrightarrow V_{s} \text { is strongly semistable. }
$$

Proof. Let $M=\oplus^{\mu} \mathcal{O}_{X}\left(1-d_{0}\right)$.

(1) By Theorem 6.3, $f_{R_{s}, I_{s}}(x)=f_{V_{s}, \mathcal{O}_{X_{s}}(1)}(x)-f_{M_{s}, \mathcal{O}_{X_{s}}(1)}(x)$. Therefore (Remark 6.6)

$$
f_{R, I}^{\infty}(x):=\lim _{p_{s} \rightarrow \infty} f_{R_{s}, I_{s}}(x)=f_{V, \mathcal{O}_{X}(1)}^{\infty}(x)-f_{M, \mathcal{O}_{X}(1)}^{\infty}(x),
$$

and hence is a continuous compactly supported function. Moreover we can write the functions $f_{V, \mathcal{O}_{X}(1)}^{\infty}$ and $f_{M, \mathcal{O}_{X}(1)}^{\infty}$ in terms of the $\mathrm{HN}$ data of $V$ and $M$ respectively. Since $\mu_{\min }(V) \leq \mu(V)<\mu(M)=\mu_{\min }(M)$ (by Remark [5.5), we have $\alpha^{\infty}(R, I)=$ $1-\mu_{\min }(V) / d$.

(2) The second assertion follows by Lemma 1.16 of [T1] which asserts that $\mu_{\min }(V)=$ $\lim _{p_{s} \rightarrow \infty} a_{\min }\left(V_{s}\right)$. 
(3) For $p_{s}>>0$ we have $a_{\min }\left(V_{s}\right) \leq \mu_{\min }\left(V_{s}\right)=\mu_{\min }(V)$ (Remark 5.5). By Theorem 6.3, $\alpha\left(R_{s}, I_{s}\right)=1-a_{\min }\left(V_{s}\right) / d$, which implies $\alpha\left(R_{s}, I_{s}\right) \geq \alpha^{\infty}(R, I)$.

If the bundle $V$ is semistable then, for $p_{s}>>0, \mu_{\min }(V)=\mu(V)=\mu\left(V_{s}\right)$. Now $\alpha\left(R_{s}, I_{s}\right)=\alpha^{\infty}(R, I)$ if and only if $a_{\min }\left(V_{s}\right)=\mu(V)$. On the other hand $a_{\min }\left(V_{s}\right)=$ $\mu\left(V_{s}\right)$ if and only if the bundle $V_{s}$ is strongly semistable on $X_{s}$.

Remark 6.8. (1) Theorem6.7(2) does not straightaway follow from Theorem6.7(1). In fact there exist uniformly convergent sequences $\left\{g_{s}: \mathbb{R}_{\geq 0} \longrightarrow \mathbb{R}_{\geq 0}\right\}_{s \in \mathbb{N}}$ consisting of compactly supported continuous functions, converging to a function $g: \mathbb{R}_{\geq 0} \longrightarrow \mathbb{R}_{\geq 0}$ but

$$
\lim _{s \rightarrow \infty} \operatorname{Sup}\left\{x \mid g_{s}(x) \neq 0\right\}>\operatorname{Sup}\{x \mid g(x) \neq 0\} .
$$

e.g., let $\left\{g_{s}:[0, \infty) \longrightarrow[0, \infty)\right\}$ be the set of functios given by

$g_{s}(x)=\frac{x}{s^{2}}$, if $x \in[0,1], \quad g_{s}(x)=\frac{2-x}{s^{2}}$, if $x \in[1,2] \quad$ and $\quad g_{s}(x)=0$, if $x \geq 2$.

(2) Note that if $I$ has homogeneous generators of degree 1, then Theorem 6.7 (2) is a consequence of Theorem 4.6 of [T4].

Corollary 6.9. Let $(R, I)$ be a pair as in Theorem 6.7. If $\operatorname{deg} \mathcal{O}_{X}(1)>2 \operatorname{genus}(X)$, where $X=\operatorname{Proj} R$, then for $p_{s}>>0$,

$$
\alpha\left(R_{s}, I_{s}\right)=\alpha^{\infty}(R, I) \Longleftrightarrow V_{s} \text { is strongly semistable. }
$$

Proof. Since, by $[\mathrm{PR}]$ and Lemma 2.1 of [T2], the bundle $V$ is semistable, the proof follows from Theorem 6.7 (3).

Proof of Theorem C and Corollary D. Theorem C and Corollary D follow from Theorem 6.7 and Corollary 6.9 (respectively) as $c^{I}(\mathbf{m})=\alpha(R, I)$, by Proposition 4.6.

Remark 6.10. We will see in the next section (Theorem 7.5) that the set of primes $p_{s}$, where the equality $c^{\mathbf{m}_{s}}\left(\mathbf{m}_{s}\right)=c_{\infty}^{\mathbf{m}}(\mathbf{m})$ holds, is always a dense (but not necessarily open) set whenever $X$ is an irreducible plane trinomial curve.

\section{F-THRESHOLDS OF PLANE TRINOMIALS}

For a pair $(R, I)$, where $R$ is a two dimensional domain and $I$ is generated by homogeneous elements of the same degree, the F-threshold $c^{I}(\mathbf{m})$ is given in terms of the minimum strong HN slope of the syzygy bundle $V$ (by Theorem B). On the other hand, if $R$ is a irreducible plane trinomial curve of degree $d \geq 3$ and $I(n)=\left(x^{n}, y^{n}, z^{n}\right)$ then all the strong HN slopes of the syzygy bundle $V$ can be computed due to a group theoretic interpretations of the complete strong HN data of the syzygy bundle (Theorem 3.5 in [T3]). Recall

$$
0 \longrightarrow V \longrightarrow \oplus \mathcal{O}_{X}(1-n) \longrightarrow \mathcal{O}_{X}(1) \longrightarrow 0
$$

is the canonical short exact sequence of $\mathcal{O}_{X}$-modules and

$$
\alpha(R, I(n))=c^{I(n)}(\mathbf{m})=1-a_{\min }(V) / d .
$$

Note that such a plane curve is either 'regular' or 'irregular' (this terminology is taken from [M2]), where a plane curve is called irregular if it has a singular point of multiplicity $r \geq d / 2$, otherwise it is called regular. 
Theorem 7.1. If $R=k[x, y, z] /(h)$, where $h$ is an irregular trinomial of degree $d$ and multiplicity $r$ (therefore $r \geq d / 2)$. Then for $\mathbf{m}=(x, y, z)$ and $I(n)=\left(x^{n}, y^{n}, z^{n}\right)$, where $n \geq 1$, we have

$$
\alpha(R, I(n))=c^{I(n)}(\mathbf{m})=\frac{n+2}{2}+\left(\frac{(2 r-d) n}{2 d}\right)^{2} .
$$

Proof. Follows from Theorem 1.1 in [T3].

For regular trinomial plane curves, we recall the following notations from [M2].

Notations 7.2. Given a regular trinomial $h$ of degree $d$ in $k[x, y, z]$ (upto linear change of variables, any such trinomial is of type I or type II, as given below), we can associate positive integers $\alpha, \beta, \nu, \lambda>0$ as follows:

(1) Type (I) $h=x^{a_{1}} y^{a_{2}}+y^{b_{1}} z^{b_{2}}+z^{c_{1}} x^{c_{2}}$, we denote $\alpha=a_{1}+b_{1}-d, \beta=a_{1}+c_{1}-d, \nu=b_{1}+c_{1}-d, \lambda=a_{1} b_{1}+a_{2} c_{2}-b_{1} c_{2}$.

(2) Type (II) $h=x^{d}+x^{a_{1}} y^{a_{2}} z^{a_{3}}+y^{b} z^{c}$, we denote

$$
\alpha=a_{2}, \beta=c, \nu=a_{2}+c-d \quad \text { and } \quad \lambda=a_{2} c-a_{3} b .
$$

Moreover we denote

$$
t_{h}=(\alpha / \lambda, \beta / \lambda, \nu / \lambda), \quad \text { and } \quad a=\operatorname{gcd}(\alpha, \beta, \nu, \lambda) \text { and } \lambda_{h}=\lambda / a .
$$

Definition 7.3. For a given regular trinomial $h$, we recall the following definition given in [HM] and [M2]. Let $L_{\text {odd }}=\left\{u=\left(u_{1}, u_{2}, u_{3}\right) \in \mathbb{Z}^{3} \mid \sum_{i} u_{i}\right.$ odd $\}$. For any $u \in L_{\text {odd }}$ and for $l, s \in \mathbb{Z}$ and $n \geq 1$, the taxicab distance

$\operatorname{Td}\left(l^{s} t_{h} n, u\right)=\operatorname{Td}\left(\left(\frac{l^{s} \alpha n}{\lambda}, \frac{l^{s} \beta n}{\lambda}, \frac{l^{s} \nu n}{\lambda}\right),\left(u_{1}, u_{2}, u_{3}\right)\right)=\left|\frac{l^{s} \alpha n}{\lambda}-u_{1}\right|+\left|\frac{l^{s} \beta n}{\lambda}-u_{2}\right|+\left|\frac{l^{s} \nu n}{\lambda}-u_{3}\right|$.

For a given regular trinomial $h$ and a given $n \geq 1$, let (1) $D_{n}(l):=s \geq 0$ if $s$ is the smallest integer, for which the inequality $\operatorname{Td}\left(l^{s} t_{h} n, u\right)<1$ has a solution for some $u \in L_{\text {odd }}$ and in that case define $T_{n}(l)=\operatorname{Td}\left(l^{s} t_{h} n, u\right)$. (2) If there is no such $s$ then we define $T_{n}(l)=1$ and $D_{n}(l)=\infty$.

We recall the following result (Theorem 3.5 in [T3]).

Theorem 7.4. For a given regular trinomial $h \in k[x, y, z]$ over a field of char $p>0$ and given $n \geq 1$, there is a well defined set theoretic map:

$$
\Delta_{h, n}: \frac{\left(\mathbb{Z} / 2 \lambda_{h} \mathbb{Z}\right)^{*}}{\{1,-1\}} \longrightarrow\left\{\frac{1}{\lambda_{h}}, \frac{2}{\lambda_{h}}, \ldots, \frac{\lambda_{h}-1}{\lambda_{h}}\right\} \times\left\{0,1, \ldots, \phi\left(2 \lambda_{h}\right)-1\right\} \bigcup\{(1, \infty)\},
$$

given by $l \rightarrow\left(T_{n}(l), D_{n}(l)\right)$, where $D_{n}(l)$ and $T_{n}(l)$ are as in Definition 7.3. Moreover

(1) $\Delta_{h, n} \equiv \Delta_{h, n+2 \lambda_{h}}$.

(2) Either $D_{n}(l)=\infty$ or $D_{n}(l)<$ the order of the element $l$ in the group $\left(\mathbb{Z} / 2 \lambda_{h} \mathbb{Z}\right)^{*}$.

Following result and explicit examples can be obtained easily from Lemma 5.4 (in [T3]).

Theorem 7.5. Let $R=k[x, y, z] /(h)$ and $\mathbf{m}=(x, y, z)$ where $h$ is a regular trinomial of degree $d$ over a field of char $p>0$. Let $\Delta_{h, n}$ be the set theoretic map given as in Theorem 7.4. Then, for $p \geq \max \left\{n, d^{2}\right\}$ and $I(n)=\left(x^{n}, y^{n}, z^{n}\right)$, where $n \geq 1$, we have the following:

(1) $p \equiv \pm 1\left(\bmod 2 \lambda_{h}\right)$ then

$$
\alpha(R, I(n))=c^{I(n)}(\mathbf{m})=\frac{n+2}{2} .
$$


(2) If $p \equiv \pm l\left(\bmod 2 \lambda_{h}\right)$ and $\Delta_{h, n}(l)=\left(T_{n}(l), D_{n}(l)\right)$ then

$$
\alpha(R, I(n))=c^{I(n)}(\mathbf{m})=\frac{n+2}{2}+\frac{\lambda\left(1-T_{n}(l)\right)}{2 p^{D_{n}(l)} d},
$$

and the integers $\lambda\left(1-T_{n}(l)\right)$ and $D_{n}(l)$ are bounded in terms of the exponents of the trinomal $h$ such that

(a) $\lambda\left(1-T_{n}(l)\right) \in\{0, \cdots, \lambda-1\}$ and

(b) $D_{n}(l) \in\{0, \ldots, O(l)\}$, where $O(l)$ is the order of the element $l$ in the group $\left.\left(\mathbb{Z} / 2 \lambda_{h} \mathbb{Z}\right)^{*}\right\}$.

Remark 7.6. Theorem 7.4 implies that for $p \geq d^{2}$ with $p \equiv \pm 1\left(\bmod 2 \lambda_{h}\right)$ (hence infinitly many primes $p$ ), we have

$$
\alpha(R, \mathbf{m})=\alpha^{\infty}(R, \mathbf{m})=c^{\mathbf{m}}(\mathbf{m})=c_{\infty}^{\mathbf{m}}(\mathbf{m})=3 / 2 .
$$

Moreover, for any given explicit trinomial $h$ and an integer $n \geq 1$, Theorem 7.4 gives an effective alogorithm to compute $\alpha(R, I(n))$ and $c^{I(n)}(\mathbf{m})$, as we need to check if the taxicab distance $\operatorname{Td}\left(l^{s} t n, u\right)<1$ has a solution for some $u \in L_{o d d}$, where $0 \leq s<\phi(2 \lambda)$ (that means for finitely many $s$ ).

Corollary 7.7. Let $S_{1}, \ldots, S_{r}$ be a set of irreducible plane trinomial curves of degrees $\geq 4$ defined over a field of characteristic 0 . Then for infinitely many primes $p=$ characteristic $k(s)$, where $s$ denotes a closed point of Spec $(A)$ we have

$$
c^{\left(\mathbf{m}_{1} \# \cdots \# \mathbf{m}_{r}\right)_{s}}\left(\left(\mathbf{m}_{1} \# \cdots \# \mathbf{m}_{r}\right)_{s}\right)=c_{\infty}^{\left(\mathbf{m}_{1} \# \cdots \# \mathbf{m}_{r}\right)}\left(\mathbf{m}_{1} \# \cdots \# \mathbf{m}_{r}\right) .
$$

If one of the trinomial is symmetric (i.e., $h=x^{a} y^{d-a}+y^{a} z^{d-a}+z^{a} x^{d-a}$, where $0 \leq$ $a \leq d)$ of degree $d \neq 5$ then there are also infinitely many primes, for which

$$
c^{\left(\mathbf{m}_{1} \# \cdots \# \mathbf{m}_{r}\right)_{s}}\left(\left(\mathbf{m}_{1} \# \cdots \# \mathbf{m}_{r}\right)_{s}\right)>c_{\infty}^{\left(\mathbf{m}_{1} \# \cdots \# \mathbf{m}_{r}\right)}\left(\mathbf{m}_{1} \# \cdots \# \mathbf{m}_{r}\right) .
$$

\section{Example 7.8.}

$$
\text { Let } \quad R=\frac{k_{p}[x, y, z]}{\left(x^{d}+y^{d}+z^{d}\right)}, \quad \mathbf{m}=(x, y, z)
$$

where $k_{p}$ denotes a field of characteristic $p \geq d^{2}$. Then by Corollary 4.4 and Theorem 4.5 of [T3]

(1) if $d>5$ is an odd integer then $p \equiv \pm(d+2)(\bmod 2 d)$ implies

$$
\alpha(R, \mathbf{m})=c^{\mathbf{m}}(\mathbf{m})=\frac{3}{2}+\frac{d-6}{2 d p} .
$$

(2) If $d \geq 4$ is an even integer then

(a) $p \equiv \pm(d+1)(\bmod 2 d)$ implies

$$
\alpha(R, \mathbf{m})=c^{\mathbf{m}}(\mathbf{m})=\frac{3}{2}+\frac{d-3}{2 d p} .
$$

(b) and $p \equiv \pm 1(\bmod 2 d)$ implies

$$
\alpha(R, \mathbf{m})=c^{\mathbf{m}}(\mathbf{m})=\frac{3}{2} .
$$

Example 7.9. Let

$$
R=\frac{k_{p}[x, y, z]}{\left(x^{d-1} y+y^{d-1} z+z^{d-1} x\right)}, \quad \text { and } \quad \mathbf{m}=(x, y, z)
$$

where $k_{p}$ denotes a field of characteristic $p \geq d^{2}$. By Corollary 4.4 and Theorem 4.5 of [T3], where $\lambda=\left(d^{2}-3 d+3\right)$ 
(1) if $p \equiv \pm 1(\bmod \lambda)$ then

$$
\alpha(R, \mathbf{m})=c^{\mathbf{m}}(\mathbf{m})=\frac{3}{2} .
$$

(2) If $p \equiv \lambda \pm 2(\bmod 2 \lambda)$ and

(a) if $d \geq 6$ is an even integer then

(i) for $3 \cdot 2^{m-2} \leq d-1<2^{m}$, where $m \geq 1$, we have

$$
\alpha(R, \mathbf{m})=c^{\mathbf{m}}(\mathbf{m})=\frac{3}{2}+\frac{2(d-2)\left(d-1-3 \cdot 2^{m-2}\right)+2}{d p^{m}},
$$

(ii) for $2^{m} \leq d-1<3 \cdot 2^{m-1}$, where $m \geq 1$, we have

$$
\alpha(R, \mathbf{m})=c^{\mathbf{m}}(\mathbf{m})=\frac{3}{2}+\frac{(d-2)\left(3 \cdot 2^{m-1}-(d-1)\right)-1}{d p^{m}} .
$$

(a) If $d \geq 7$ is an odd integer then

$$
\alpha(R, \mathbf{m})=c^{\mathbf{m}}(\mathbf{m})=\frac{3}{2}+\frac{\lambda-6(d-2)}{2 d p} .
$$

(b) If $d=5$ then

$$
\alpha(R, \mathbf{m})=c^{\mathbf{m}}(\mathbf{m})=\frac{3}{2}+\frac{7}{2 d p^{3}} .
$$

\section{REFERENCES}

[AHN] Àlvarez Montaner, J., Huneke, C., Núñez-Betancourt, L., D-modules, BernsteinSato polynomials and F-invariants of direct summands, Advances in Mathematics Volume 321, 1 December 2017, Pages 298-325.

[BMS1] Blickle, M., Mustaţă, M., Smith, K., F-thresholds of hypersurfaces, Trans. Amer. Math. Soc. 361 (2009), no. 12, 6549-6565.

[BMS2] Blickle, M., Mustaţă, M., Smith, K.,Discreteness and rationality of F-thresholds, Michigan Math. J., 57 (2008), pp. 43-61 (Special volume in honor of Melvin Hochster).

[CM] Chiba, T., Matsuda, K., Diagonal F-thresholds and F-pure thresholds of Hibi rings, Comm. Algebra 43 (2015), no. 7, 2830-2851.

[DsNbP] Stefani, A., Núñez-Betancourt, L., Prez, F., On the existence of F-thresholds and related limits, Trans. Amer. Math. Soc. 370 (2018), no. 9, 6629-6650.

[EGA IV] Grothendieck, A., Dieudonné, J.A., Eléments de Géometrie Algébrique IV, Pub. Math. IHÉS.

[G] Gieseker, D., Stable vector bundles and the Frobenius morphism, Ann. Sci. cole Norm. Sup. (4) 6 (1973), 95-101.

[HM] Han, C., Monsky, P., Some surprising Hilbert-Kunz functions, Math. Z., 214 (1993), no. 1, 119135.

[H] Hara, N. F-pure thresholds and F-jumping exponents in dimension two, Math.Res. Lett. 13 (2006), no. 56, 747-760. With an appendix by Paul Monsky.

[HL] Huybrechts, D., Lehn, M., The geometry of moduli spaces of sheaves, Aspects of Mathematics, E31. Friedr. Vieweg \& Sohn, Braunschweig, 1997.

[HY] Hara, N., Yoshida, K., A generalization of tight closure and multiplier ideals, Trans. Am. Math. Soc. 355, 3143-3174 (2003).

[HWY] Hirose, D., Watanabe, K.I., Yoshida, K.I., F-thresholds versus a-invariants for standard graded toric rings, Comm. Algebra, 42 (6) (2014), pp. 2704-2720.

[HH1] Hochster, M., Huneke, C. Tight closure, invariant theory and the Briancon-Skoda theorem, J. Amer. Math. Soc. 3 (1990), 31-116.

[HH2] Hochster, M., Huneke, C. F-regularity, test elements, and smooth base change, Trans. Amer. Math. Soc. 346 (1994), no. 1, 1-62.

[HMTW] Huneke, C., Mustaţă, M., Takagi, S., Watanabe, K.I., F-thresholds, tight closure, integral closure and multiplicity bounds Michigan Math. J. 57, in Special Volume in Honor of Melvin Hochster, Univ. Michigan Press, Ann Arbor, (2008), 463-483. 
[KLZ] Katzman, M., Lyubeznik, G., Zhang, W., On the discreteness and rationality of F-jumping coefficients, J. Algebra 322 (2009), no. 9, 3238-3247.

[L] Langer, A., Semistable sheaves in positive characteristic, Ann. Math., 159 (2004).

[M1] Monsky, P., The Hilbert-Kunz function, Math. Ann. 263 (1983) 43-49.

[M2] Monsky, P., The Hilbert-Kunz multiplicity of an irreducible trinomial, Journal of Algebra 304 (2006) 1101-1107.

[Ma] Maruyama, M., Openness of a family of torsion free sheaves, J. Math.Kyoto Univ., 16-3 (1976), 627-637.

[MTW] Mustaţă, M., Takagi, S., Watanabe, K.I., F-thresholds and Bernstein-Sato polynomials, European congress of mathematics, 341-364, Eur. Math. Soc., Zurich, 2005.

[PR] Paranjape, K., Ramanan, S., On the canonical rings of a curve, Algebraic Geometry and Commutative Algebra, Vol. II (Kinokunia,Tokyo), (1968), 503-616.

[S] Smith, K., Tight closure in graded rings, J.Math.Kyoto Univ. 37 (1997), no. 1, 35-53.

[TW] Takagi, S., Watanabe, K.I., On F-pure thresholds, J. Algebra 282 (2004), 278-297.

[T1] Trivedi, V., Hilbert-Kunz multiplicity and reduction mod p, Nagoya Math. Journal !85 (2007), 123-141.

[T2] Trivedi, V., Hilbert-Kunz density Function and Hilbert-Kunz multiplicity, Trans. Amer. Math. Soc. 370 (2018), no. $12,8403-8428$.

[T3] Trivedi, V., Arithmetic behaviour of Frobenius semistability of syzygy bundles for plane trinomial curves, Bull. Sci. Math. 141 (2017), no. 7, 703-726.

[T4] Trivedi, V., Towards Hilbert-Kunz density functions in characateristic 0, Nagoya Math. J., 235 (2019), 158-200.

[T5] Trivedi, V., Nondiscreteness of F-thresholds, To appear in Math Research letters.

[Vr] Vraciu, A., On the degrees of relations on $x_{1}^{d_{1}}, \ldots, x_{n}^{d_{n}},\left(x_{1}+\cdots+x_{n}\right)^{d_{n+1}}$ in positive characteristic, J. of Algebra 423 (2015), 916-949.

School of Mathematics, Tata Institute of Fundamental Research, Homi Bhabha Road, MumBAI-40005, INDIA

E-mail address: vija@math.tifr.res.in

Department of Mathematics, College of Humanities and Sciences, Nihon University, Setagaya-Ku, TOKYO 156-0045, JAPAN

E-mail address: watanabe@math.chs.nihon-u.ac.jp 